\title{
Investigating the Influence of Tracer Kinetics on Competition-Kinetic Association Binding Assays: Identifying the Optimal Conditions for Assessing the Kinetics of Low-Affinity Compounds \$
}

\author{
David A. Sykes, Palash Jain, and Steven J. Charlton \\ School of Life Sciences, Queen's Medical Centre, University of Nottingham, Nottingham, United Kingdom (D.A.S., P.J., S.J.C.); \\ Centre of Membrane and Protein and Receptors (COMPARE), University of Birmingham and University of Nottingham, Midlands, \\ United Kingdom (D.A.S., P.J., S.J.C.); and Excellerate Bioscience Ltd, Discovery Building, BioCity, Nottingham, United Kingdom \\ (S.J.C.)
}

Received March 23, 2019; accepted June 22, 2019

\begin{abstract}
An increased appreciation of the importance of optimizing drugbinding kinetics has lead to the development of various techniques for measuring the kinetics of unlabeled compounds. One approach is the competition-association kinetic binding method first described in the 1980s. The kinetic characteristics of the tracer employed greatly affects the reliability of estimated kinetic parameters, a barrier to successfully introducing these kinetic assays earlier in the drug discovery process. Using a modeling and Monte Carlo simulation approach, we identify the optimal tracer characteristics for determining the kinetics of the range of unlabeled ligands typically encountered during the different stages of a drug discovery program (i.e., rapidly dissociating, e.g., $k_{\text {off }}=10$ minute $^{-1}$ low-affinity "hits" through to slowly dissociating e.g., $k_{\text {off }}=0.01$ minute $^{-1}$ high-affinity "candidates"). For more rapidly dissociating ligands (e.g., $k_{\text {off }}=$
\end{abstract}

10 minute $\left.^{-1}\right)$, the key to obtaining accurate kinetic parameters was to employ a tracer with a relatively fast off-rate (e.g., $k_{\text {off }}=$ 1 minute $^{-1}$ ) or, alternatively, to increase the tracer concentration. Reductions in assay start-time $\leq 1$ second and read frequency $\leq 5$ seconds significantly improved the reliability of curve fitting. Timing constraints are largely dictated by the method of detection, its inherent sensitivity (e.g., TR-FRET versus radiometric detection), and the ability to inject samples online. Furthermore, we include data from TR-FRET experiments that validate this simulation approach, confirming its practical utility. These insights into the optimal experimental parameters for development of competition-association assays provide a framework for identifying and testing novel tracers necessary for profiling unlabeled competitors, particularly rapidly dissociating lowaffinity competitors.

\section{Introduction}

Historically, most drug discovery programs have relied upon equilibrium dissociation constant measurements when assessing the potential of future lead-drug candidates, rather than the kinetic parameters that comprise it $\left(K_{\mathrm{d}}=k_{\mathrm{off}} / k_{\mathrm{on}}\right)$. However, it is becoming more widely appreciated that optimizing the kinetics of drug binding $\left(k_{\text {off }}\right.$ and $\left.k_{\text {on }}\right)$ can enhance both compound efficacy and duration of action (Sykes et al., 2009; Guo et al., 2012; Copeland, 2016; Vauquelin, 2016). This has resulted in the introduction of novel methods to assess ligand binding kinetics at earlier stages in the drug discovery process, allowing the development of structure-kinetic relationships (Schmidtke et al., 2011; Georgi et al., 2018). A commonly applied method to assess the kinetics of unlabeled compounds is surface plasmon resonance (SPR) spectroscopy,

https://doi.org/10.1124/mol.119.116764.

S This article has supplemental material available at molpharm. aspetjournals.org. but this is still not widely used for membrane proteins due to issues with protein purification and stability. To overcome this, competition-association kinetic binding assays using radioactive probes have been employed successfully to determine the kinetic values for both antagonists (Gillard and Chatelain, 2006; Slack et al., 2011; Fleck et al., 2012; Sykes et al., 2014, 2016) and agonists (Sykes et al., 2009; Sykes and Charlton, 2012) acting at a variety of G protein-coupled receptors at physiologic temperature and even more recently at non-G protein-coupled receptors targets (Yu et al., 2015).

Despite their undoubtable high sensitivity, the use of radioactive probes as tracers in kinetic competition binding assays presents a number of challenges. Classic radioactive binding requires the need for multiple washing steps to separate bound and free radioligand, adding complexity to the procedure and reducing throughput. Importantly, the wash step requirement also prevents any possibility of multiple single sample reads. More recently, scintillation

ABBREVIATIONS: HTRF, homogeneous time resolved fluorescence; PPHT-red, ( \pm )-2-(n-phenethyl-n-propyl)amino-5-hydroxytetralin hydrochloride;1-naphthalenol, 5,6,7,8-tetrahydro-6-[(2-phenylethyl)propylamino]) derivative labeled with a red fluorescent probe; SPR, surface plasmon resonance; TR-FRET, time-resolved fluorescent resonance energy transfer. 
proximity assays have been formulated that can be performed in homogeneous conditions, but signal-drift due to bead settling can complicate interpretation at early time points (Xia et al., 2016).

The emergence of time-resolved fluorescent resonance energy transfer (TR-FRET)-based methods (Schiele et al., 2015; Klein-Herenbrink et al., 2016) and bioluminescence based methods (Stoddart et al., 2018; Bouzo-Lorenzo et al., 2019) offers an alternative to radioactive binding assays and represents higher throughput methods to assess unlabeled ligand kinetics. With separation of bound and unbound label no longer necessary, multiple reads can be made from the same well (Emami-Nemini et al., 2013; Stoddart et al., 2015). Using the simple technique of TR-FRET we successfully characterized the binding of a series of dopamine $\mathrm{D}_{2}$ specific agonists and clinically used antipsychotics employing a SNAP-tagged receptor labeled with terbium and a fluorescent tracer (Klein-Herenbrink et al., 2016; Sykes et al., 2017).

A key observation made during these studies was that the kinetic characteristics of the tracer had a profound effect on the reliability of the estimated kinetic parameters of unlabeled competitors (Klein-Herenbrink et al., 2016). This was most evident when using a slowly dissociating tracer to assess rapidly dissociating, low-affinity ligands. This represents a potential barrier to introducing successfully kinetic assays earlier in the drug discovery process (e.g., hit identification/ validation) as tracers need to be capable of determining the kinetics of low-affinity "hits" (in the micromolar range). Thus the aim of the current study was to use a modeling and simulation approach to identify the optimal tracer characteristics for determining the kinetics of a variety of unlabeled ligands typically encountered during different stages of a drug discovery program (i.e., low-affinity "hits" through to highaffinity "candidates").

Using Monte Carlo simulations, we have assessed the ability of the global association method to accurately determine the kinetics of different model tracers. The global association method is a way of simultaneously fitting multiple tracer association curves (e.g., different tracer concentrations) to find best-fit $k_{\text {off }}$ and $k_{\text {on }}$ parameters across the whole dataset. Additionally, using Monte Carlo simulations we have explored the utility of these model tracers to predict the kinetic parameters of unlabeled ligands in competitionassociation binding mode. Furthermore, we have explored the influence of experimental design on the accuracy of kinetic parameter estimation, testing the impact of increasing tracer concentrations and investigating different plate reader configurations, particularly the influence of read frequency time and on-line (direct reagent injection into sample wells) versus off-line (reagent addition into plate prior to insertion into reader) reagent addition.

Finally we test the validity of this modeling approach through the detailed characterization of two dopamine $\mathrm{D}_{2} \mathrm{R}$ specific fluorescent tracers, spiperone-d2 and PPHT-red, assessing their ability to determine the kinetics of $D_{2} R$ specific ligands with very varied kinetic characteristics, from the rapidly dissociating chlorpromazine to the very slowly dissociating butaclamol.

The analysis presented has important implications for the design of competition-kinetic approaches to assess unlabeled compound kinetics, providing a framework for the identification and testing of suitable tracers. In particular, we have identified the optimal tracer characteristics and experimental design for assessing low-affinity competitors, enabling the utilization of kinetics assays much earlier in the drug discovery process.

\section{Materials and Methods}

\section{Determining the Kinetics of the Tracer for Use in Competition Kinetic Binding Studies}

Monte Carlo simulations are useful to investigate the behavior of a system under controlled situations and may be thought of as statistical experiments. They provide an estimation of variance for complex models, which ultimately helps researchers with experimental design and provides confidence in a particular experimental approach prior to testing. The underlying principle is to take a simulated dataset that is based on a set of "ideal" model parameters, add random error to the "dataset," and then recreate the resulting dataset many times to obtain the parameters of interest. In this case the "dataset" comprises an $\mathrm{XY}$ table where $\mathrm{X}$ is time and $\mathrm{Y}$ is percentage of specific binding of the tracer. This process allows a frequency distribution to be built from the resulting parameter estimates, allowing an understanding of the associated error of each parameter estimate under the conditions employed (Christopoulos, 2001).

The competition association assay model (Motulsky and Mahan, 1984) relies on an accurate assessment of the kinetic properties of the labeled tracer, both the association rate constant $\left(k_{\text {on }}\right.$ or $\left.k 1\right)$ and the dissociation rate constant $\left(k_{\text {off }}\right.$ or $\left.k 2\right)$. Since the introduction of fitting software, such as GraphPad Prism (San Diego, CA), a popular experimental procedure to estimate the kinetics of the tracer is to monitor the binding of two or more different concentrations of tracer over time until equilibrium is reached; in doing so it is possible to calculate the $k_{\text {on }}$ and $k_{\text {off }}$ values of the tracer by simultaneously fitting all curves to generate global estimates for these rate parameters.

For the purposes of this study, tracer association was simulated using GraphPad Prism 6.0 employing the following equation, where $k_{\text {obs }}$ equals the observed rate of association and $k_{\text {on }}$ and $k_{\text {off }}$ are the association and dissociation rate constants, respectively, of the tracer:

$$
\begin{gathered}
k_{\mathrm{ob}}=[\mathrm{L}] * k_{\mathrm{on}}+k_{\mathrm{off}} \\
Y=\mathrm{Y}_{\max } *\left(1-\exp \left(-1 * k_{\mathrm{ob}} * \mathrm{X}\right)\right)
\end{gathered}
$$

In this globally fitted model of tracer binding, tracer concentrations ([L]) are fixed and $k_{\text {on }}$ and $k_{\text {off }}$ are shared parameters and independent of tracer concentration. Here, $\mathrm{Y}$ is the level of receptor-bound tracer, $\mathrm{Y}_{\max }$ is the level of tracer binding at equilibrium, $\mathrm{X}$ is in units of time (e.g., minutes), and $k_{\text {obs }}\left(\right.$ minute ${ }^{-1}$ ) is the rate in which equilibrium is approached.

Tracer binding simulations were performed to assess the effect of on-line and off-line reagent addition on our ability to estimate accurately the kinetics of a variety of model tracers with varied kinetics parameters, consistent with the properties of compounds discovered in the various phases of the drug discovery cascade, the details of which are provided in Table 1. Tracer Monte Carlo simulations were performed using the following parameters: the $k_{\text {on }}$ and $k_{\text {off }}$ of each model tracer studied were allowed to vary, while the concentrations of tracer ([L]) employed were fixed at various multiples of the tracer's affinity, specifically $30,10,3,1,0.3,0.1 \times K_{\mathrm{d}}$. Unless otherwise stated, the assay start time was fixed at either 1 second to mimic on-line addition of reagents via plate reader injectors or 30 seconds to mimic the delay in the time to read following off-line addition of membranes prior to insertion the assay plate into the plate reader. Read frequency (i.e., the time between each well read) was varied at $1,5,10,20$, and 60 seconds. Random error was added to the generated Y values by taking each theoretical (i.e., "correct") value and 
TABLE 1

Equilibrium affinity and kinetic properties of the tracer compounds used to construct Figs. 1-9 and Supplemental Figs. 1-7 and typical of the kinetic parameters of compounds discovered at all phases of the drug discovery screening cascade

\begin{tabular}{lccl}
\hline Tracer $K_{\mathrm{D}}$ & Tracer $k_{\text {off }}$ & Tracer $k_{\text {on }}$ & \multicolumn{1}{c}{ Characteristic of Tracer } \\
\hline$n M$ & min $^{-1}$ & $M^{-1} \cdot \mathrm{min}^{-1}$ & \\
0.01 & 0.01 & $1 \times 10^{9}$ & Very high-affinity candidate, very slow off \\
1 & 0.1 & $1 \times 10^{8}$ & High-affinity candidate, slow off \\
100 & 1 & $1 \times 10^{7}$ & Lead-like, fast off \\
300 & 10 & $3 \times 10^{7}$ & Hit-like, very fast off \\
\hline
\end{tabular}

adding to it a random number taken from a uniformly distributed population with an S.D. equal to one. The random error chosen for simulation was Gaussian absolute to reflect directly the pattern of error observed in our experimental data. The resulting simulated datasets were then individually fit to the association kinetic model (two or more concentrations of hot, GraphPad Prism 6.0) and "Global (shared) parameters" including tracer $k_{\text {off }}$ and $k_{\text {on }}$ values were tabulated. In total, 200 simulations were performed per test condition.

\section{Determining the Suitability of the Tracer for Competition Kinetic Binding Studies}

The interactions of the tracer and unlabeled competitor with receptor is described by two differential equations that, when solved, yield a single equation describing the binding of the tracer as a function of time (Motulsky and Mahan, 1984). This model was used to simulate competition association curves to a variety of unlabeled compounds with different association-rate constants $\left(k_{\text {on }}\right.$ or $\left.k 3\right)$ and dissociationrate constants ( $k_{\text {off }}$ or $\left.k 4\right)$, according to the following equations:

$$
\begin{gathered}
K_{A}=k_{1}[L]+k_{2} \\
K_{B}=k_{3}[I]+k_{4} \\
S=\sqrt{\left(\left(K_{A}-K_{A}\right)^{2}+4 \cdot k_{1} \cdot k_{3} \cdot[L] \cdot[I] \cdot 10^{-18}\right)} \\
K_{F}=0.5 \cdot\left(K_{A}+K_{B}+S\right) \\
K_{S}=0.5 \cdot\left(K_{A}+K_{B}-S\right) \\
D I F F=K_{F}-K_{S} \\
Q=\frac{B_{\max } \cdot K_{1} \cdot[L] \cdot 10^{-9}}{D I F F} \\
Y=Q \cdot\left(\frac{k_{4} \cdot D I F F}{K_{F} \cdot K_{S}}+\frac{k_{4}-K_{F}}{K_{F}} \cdot \exp ^{\left(-K_{F} \cdot X\right)}-\frac{k_{4}-K_{S}}{K_{S}} \cdot \exp ^{\left(-K_{S} \cdot X\right)}\right)
\end{gathered}
$$

where $\mathrm{X}=$ time (minutes), $\mathrm{Y}=$ specific binding (e.g., cpm or HTRF units), $k_{1}=k_{\text {on }}$ tracer $\left(\right.$ molar $^{-1} \cdot$ minute $\left.^{-1}\right), k_{2}=k_{\text {off }}$ tracer $\left(\right.$ minute $\left.{ }^{-1}\right)$, $[L]=$ concentration of tracer used (nanomolars), $[I]=$ concentration unlabeled ligand (nanomolars). Fixing the above parameters allows the following to be calculated: $k_{3}=$ association-rate constant of unlabeled ligand $\left(\mathrm{molar}^{-1} \cdot\right.$ minute $\left.^{-1}\right), k_{4}=$ dissociation-rate constant of unlabeled ligand (minute ${ }^{-1}$ ), $B_{\max }=$ maximal specific binding of the system at equilibrium binding (e.g., cpm or HTRF units) (Motulsky and Mahan, 1984).

All competition kinetic association simulations were performed using the model tracers described in Table 1. These Monte Carlo simulation studies were designed to assess the effect of tracer kinetics on the ability of the Motulsky-Mahan model to distinguish compounds typically encountered in a drug discovery program with a wide range of kinetic off-rates ranging from the more rapidly dissociating (e.g., $k_{\text {off }}$ $=100$ minute $^{-1}$ representative of low-affinity fragments) to the more slowly dissociating (e.g., $k_{\text {off }}=0.01$ minute $^{-1}$ representative of a lead candidate compound).

Unless otherwise stated, the concentration of tracer ([L]) employed was fixed at $3 \times K_{\mathrm{d}}$. The concentration of unlabeled competitor ([I]) was routinely fixed at various multiples of its own affinity, specifically 100 ,
$30,10,3,1 \times K_{\mathrm{d}}$. Also taken into consideration were on-line versus offline addition protocols and their effect on our ability to accurately estimate the kinetics of unlabeled compounds with varied kinetics parameters. Start time was fixed at either 1 second (to mimic on-line addition) or 30 seconds (to mimic off-line addition), and the read frequency was varied using either $1,5,10,20$, or 60 seconds unless otherwise stated.

Random error was added to the generated $Y$ values by taking each theoretical (i.e., "correct") value and adding to it a random number taken from a uniformly distributed population with an S.D. equal to one. The random error chosen for simulation was Gaussian absolute to directly reflect the pattern of error observed in our experimental data. The resulting simulated datasets were then individually fit to the competition association model (kinetics of competitive binding, GraphPad Prism 6.0) model and "Global (shared) parameters" including tracer $k_{\text {off }}$ and $k_{\text {on }}$ values were tabulated. In total, 200 simulations were performed per test condition.

To test the effect of tracer concentration on our ability to estimate the kinetic parameters of unlabeled compounds, we performed a further series of simulations, but this time we employed tracer concentrations ([L]) at $1 \times, 10 \times$, and $30 \times K_{\mathrm{d}}$. The concentrations of unlabeled competitor ([I]) were varied accordingly. This allowed us to test the effect of decreasing and increasing tracer competition on the accuracy of unlabeled compound kinetic determinations.

In another series of competition kinetic association simulations, the ability of a rapidly dissociating tracer to assess the kinetics of low affinity $(1-1000 \mu \mathrm{M})$ unlabeled fragments was explored in both double and single concentration screening modes. All Monte Carlo simulations were performed using the following parameters: the $k_{\text {on }}$ and $k_{\text {off }}$ of the model tracer studied was fixed at $3 \times 10^{7} \mathrm{M}^{-1}$ minute ${ }^{-1}$ and 10 minute $^{-1}$, respectively, with the concentration of tracer ([L]) employed fixed at $3 \times K_{\mathrm{d}}$, while the concentrations of the fragments were fixed specifically at 10 and $100 \mu \mathrm{M}$ (typical of a fragment screen where affinity of the fragments is unknown). Start time was fixed at 1 second (to mimic on-line addition), and a standard read frequency of 5 seconds was employed. The resulting simulated datasets were then individually fit to the competition association model and "Global (shared) parameters" including tracer $k_{\text {off }}$ and $k_{\text {on }}$ values were tabulated. In total, 200 simulations were performed per test condition. All the experiments described above assume that the Laws of Mass Action are obeyed and that ligand depletion does not occur under any of the conditions of tracer and competitor binding explored (Carter et al., 2007).

\section{Monte Carlo Simulation Data Analysis}

All Monte Carlo simulations were performed in Prism 6.0. In total, 200 simulations were performed per test condition using an associated error equivalent to an S.D. of one. "Global (shared) parameters" associated with eqs. 1 and 2 were recorded, and an outlier test (using an iterative Grubb's test, $\alpha=0.0001$ ) was performed on reported $k_{\text {on }}$ and $k_{\text {off }}$ values for tracers and unlabeled competitors. Outliers and ambiguous fits (those for which confidence intervals were extremely wide) were removed from the analysis before calculation of mean and $\%$ coefficient of variance (CV) values [i.e., (S.D./mean)*100], which are indications of accuracy and variation around the expected input value. Mean fitting values were considered a reliably "good fit" if $>90 \%$ of fits 
(i.e., $>180 / / 200$ fits) were returned without being judged as either outliers and/or ambiguous fits. Ambiguous fits are those for which confidence intervals are extremely wide and therefore not reported (http://www.graphpad.com/guides/prism/7/curve-fitting/index.htm). Graphical representation of Monte Carlo data was also performed in using R, and competition association data were fitted in Prism 6.0.

\section{Fluorescent Ligand Binding Assays}

Materials. PPHT (( \pm$)-2$-( $n$-phenethyl- $n$-propyl)amino-5-hydroxytetralin hydrochloride;1-naphthalenol, 5,6,7,8-tetrahydro-6-[(2-phenylethyl) propylamino]) derivative labeled with a red fluorescent probe (PPHTred) was obtained from Cisbio Bioassays (Bagnolssur-Cèze, France). Ninety-six-well polypropylene plates (Corning) were purchased from Fisher Scientific UK (Loughborough, UK), and 384-well optiplate plates were purchased from PerkinElmer (Beaconsfield, UK). GppNHp, risperidone, chlorpromazine hydrochloride, and (+)-butaclamol used in competition assays were obtained from Sigma-Aldrich (Poole, UK). Domperidone and haloperidol hydrochloride used for competition assays were obtained from Tocris Bioscience (Avonmouth, Bristol). Pergolide and ropinirole were kind gifts from Dr. Robert Lane (Monash University, Melbourne, Australia), while bromocriptine was a kind gift of Dr. Nicholas Holliday (Nottingham University, Nottingham, UK).

Determination of $D_{2} R$ fluorescent ligand binding kinetics. All fluorescent binding experiments using PPHT-red and spiperone-d2 were conducted in white 384-well Optiplate plates in assay binding buffer, Hanks' balanced salt solution containing $5 \mathrm{mM}$ HEPES, $1 \% \mathrm{DMSO}$, and $0.02 \%$ pluronic acid $\mathrm{pH} 7.4$, and $0.1 \mathrm{mM}$ GppNHp. GppNHp was included to remove the $\mathrm{G}$ protein-coupled population of receptors that can result in two distinct populations of binding sites in membrane preparations, since the Motulsky-Mahan model is only appropriate for ligands competing at a single site. In all cases, nonspecific binding was determined in the presence of $10 \mu \mathrm{M}$ haloperidol.

To accurately determine association rate $\left(k_{\text {on }}\right)$ and dissociation rate $\left(k_{\text {off }}\right)$ values, the observed rate of association $\left(k_{\text {obs }}\right)$ was calculated using at least four different concentrations of either PPHT-red or spiperone-d2. SNAP-tagged terbium-labeled human dopamine $\mathrm{D}_{2 \mathrm{~L}}$ receptors expressed in CHO cell membranes ( $2 \mu \mathrm{g} /$ well) were injected into wells containing six different concentrations of the fluorescent tracers PPHT-red (50-1.56 nM) or spiperone-d2 (10-0.3 nM) in a final assay volume of $40 \mu \mathrm{l}$. A detailed description of the terbium labeling procedure can be found in Sykes et al. (2017).

The degree of PPHT-red or spiperone-d2 bound to the receptor was assessed at multiple time points by HTRF detection to allow construction of association kinetic curves. The resulting data were globally fitted to the association kinetic model (eq. 1) to derive a single best-fit estimate for $k_{\text {on }}$ and $k_{\text {off- }}$

Competition binding kinetics. To determine the association and dissociation rates of $\mathrm{D}_{2} \mathrm{R}$ ligands, we used a competition kinetic binding assay we recently described to profile the kinetics of a series of $\mathrm{D}_{2} \mathrm{R}$ agonists (Klein-Herenbrink et al., 2016). This approach involves the addition of a receptor preparation to wells containing fluorescent ligand and competitor, so that at $t=0$ all receptors are unoccupied.

PPHT-red (12.5 nM; $1 \times K_{\mathrm{d}}$, a concentration that avoids ligand depletion in this assay volume; see Carter et al., 2007) was added simultaneously with the unlabeled compound (at $t=0$ ) to CHO cell membranes containing the human dopamine $\mathrm{D}_{2 \mathrm{~L}}$ receptor $(2 \mu \mathrm{g} / \mathrm{well})$ in $40 \mu \mathrm{l}$ of assay buffer.

On-line addition is best achieved by injecting an equal volume of receptor (20 $\mu \mathrm{l}$ membranes) to the assay plate containing the ligands of interest (e.g., tracer and/or competitor $20 \mu \mathrm{l}$ ) to ensure complete and adequate mixing of all reagents. The degree of PPHT-red bound to the receptor was assessed at multiple time points by HTRF detection. The kinetic parameters of PPHT-red and spiperone-d2 plus those of unlabeled compounds were determined using a start time of $\sim 1$ second and a read frequency of 5 seconds.
Nonspecific binding was determined as the amount of HTRF signal detected in the presence of haloperidol $(10 \mu \mathrm{M})$ and was subtracted from each time point, meaning that $t=0$ was always equal to zero. Each time point was conducted on the same 384-well plate incubated at room temperature with orbital mixing ( 1 second of $100 \mathrm{rpm} /$ cycle).

Data were globally fitted using eq. 2 to simultaneously calculate $k_{\text {on }}$ and $k_{\text {off }}$ of the unlabeled compounds. Different competitor concentration ranges were chosen, as compounds with a long residence time equilibrate more slowly, so a higher relative concentration is required to ensure the experiments reach equilibrium within a reasonable time frame (20 minutes), while still maintaining a good signal-to-noise.

\section{Signal Detection}

For the binding experiments described, signal detection was performed on a Pherastar FS (BMG Labtech, Offenburg, Germany) using standard HTRF settings. The terbium donor was always excited with up to six laser flashes at a wavelength of $337 \mathrm{~nm}$. A kinetic TRFRET signal was collected at 5-second intervals at both 665 and 620 $\mathrm{nm}$ when using red acceptor. HTRF ratios were obtained by dividing the acceptor signal $(665 \mathrm{~nm})$ by the donor signal $(620 \mathrm{~nm})$ and multiplying this value by 10,000 .

\section{Results}

\section{Monte Carlo Simulations}

Accurate Determination of Model Tracer Kinetic Parameters. To accurately calculate the kinetic on $\left(k_{\text {on }}\right)$ and off rates $\left(k_{\text {off }}\right)$ of unlabeled competitor compounds, the kinetic parameters of the tracer used must first be determined by fitting tracer association data to a global kinetic model (see eq. 1). Simulations were performed for four model tracers whose rates of dissociation differed up to 1000-fold (see Table 1).

For each tracer tested, a family of association kinetic curves were constructed using six concentrations of each tracer, ranging from $\sim 30$ to $0.1 \times K_{\mathrm{d}}$, with each association curve being monitored to the point of equilibrium, such that $\mathrm{Y}_{\max }$ is reached, allowing for the most accurate estimation of tracer kinetic parameters, $k_{\text {on }}$ and $k_{\text {off }}$ (see Fig. 1 , A and $\mathrm{C}$ ).

To construct Fig. 1, A and C, using Monte Carlo simulations, the $k_{\text {on }}$ input value for the very rapidly dissociating tracer was fixed at $3 \times 10^{7} \mathrm{M}^{-1}$ minute $^{-1}$ and $k_{\text {off }}$ input value at 10 minute $^{-1}$. Figure 1, $\mathrm{A}$ and $\mathrm{C}$, differ only in their initial start time 1 (representative of on-line injection) and 30 seconds (representative of off-line addition), respectively, with read frequency fixed every 10 seconds thereafter. What is immediately apparent under these simulation conditions is that the equilibrium between a very rapidly dissociating tracer $\left(k_{\text {off }}\right.$ 10 minute $^{-1}$ ) and receptor is reached rapidly within the first 30 second, and, as a consequence, an accurate determination of tracer association is improbable with a 30 -second start time. This is reflected in the graphical plots, Fig. 1, B and D, showing the relationship between input tracer $k_{\text {off }}$ and output $k_{\text {off }}$ with read frequency. With a start time of 1 second, tracer kinetic determinations (data fits) are on the whole extremely reliable, with a "good fit" achieved on $>90 \%$ of the 200 fits (see Fig. 1, B and C, and Supplemental Table 1). In contrast, reliable determinations of tracer kinetics, as judged through off-rate monitoring, following a 30-second start time representative of off-line addition, are only possible when the offrate of the tracer is less than 1 minute $^{-1}$.

Also apparent is the effect of read frequency on the variation in reported $k_{\text {off }}$ values with shorter read frequencies, resulting 


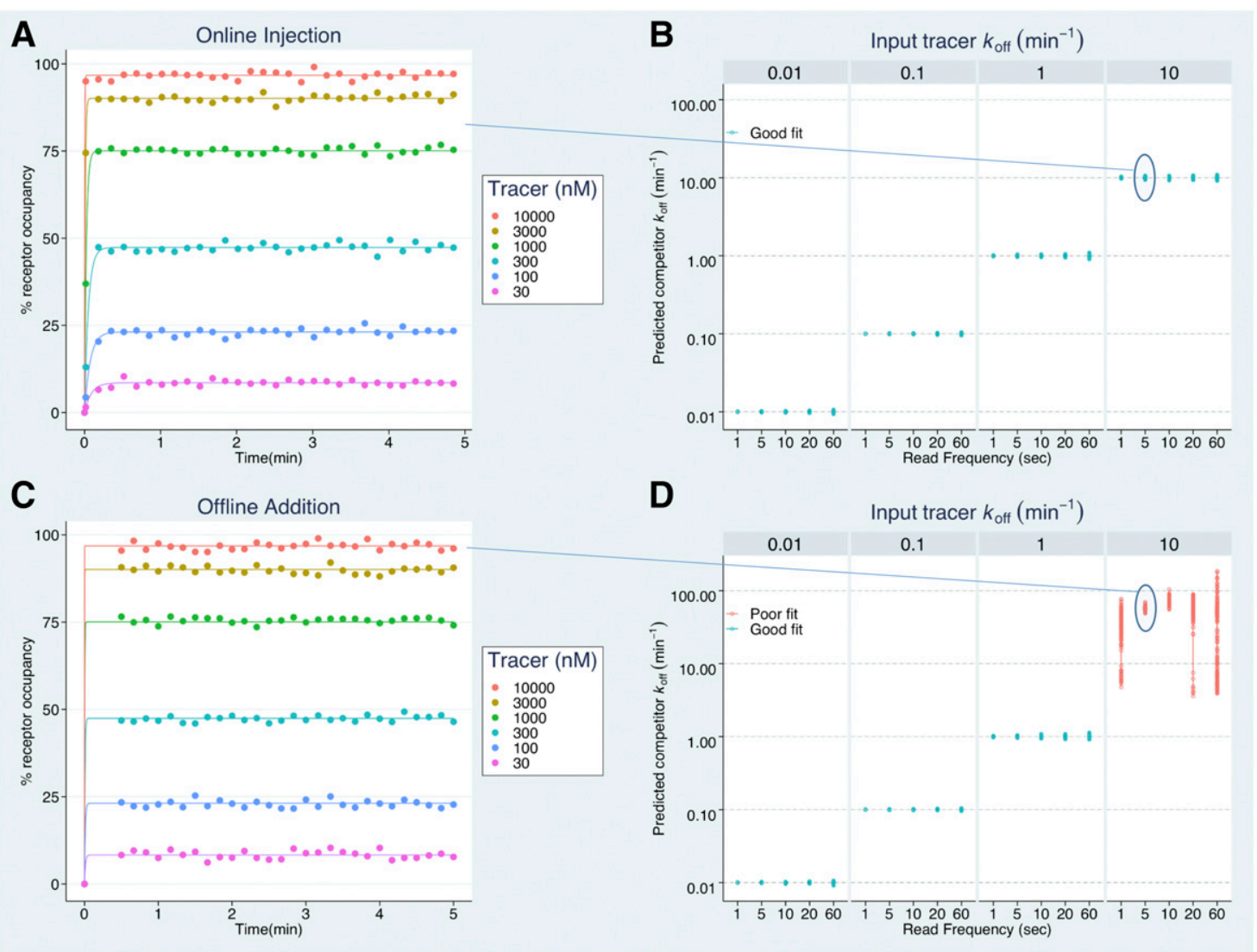

Fig. 1. Monte Carlo simulation results exploring the effect of sample time on the determination of tracer kinetic parameters. (A) Association with time (1-second start; 10 -second interval) of various concentrations of a very rapidly dissociating tracer with the following kinetic parameters; $k_{\text {off }}$ of 10 minute ${ }^{-1}$ $k_{\text {on }}$ of $3 \times 10^{7} \mathrm{M}^{-1}$.minute ${ }^{-1}$ representative of 200 simulations. In this globally fitted model (see eq. 1) of tracer binding, tracer concentrations are fixed, $k_{\text {on }}$ and $k_{\text {off }}$ are shared parameters to be determined. (B) Effect of read frequency on the output $k_{\text {off }}$ of a series of experimental tracers with varied kinetics, absolute values are the average of 200 simulations. In this instance, tracer simulations were performed with an initial start time of 1 second, representative of injection of receptor to a reaction containing only tracer. Blue symbols represent conditions that returned $>90 \%$ reliable fits. Red symbols represent conditions that returned $<90 \%$ reliable fits. (C) Association with time (30-second start; 10 -second interval) of various concentrations of a very rapidly dissociating tracer with the following kinetic parameters; $k_{\text {off }}$ of 10 minute ${ }^{-1}, k_{\text {on }}$ of $3 \times 10^{7} \mathrm{M}^{-1} \cdot$ minute $^{-1}$ representative of 200 simulations. (D) Effect of read frequency on the output $k_{\text {off }}$ of a series of experimental tracer with varied kinetics. In this instance tracer simulations were performed with an initial start time of 30 second representative of off-line addition of receptor to a reaction containing only tracer, absolute values are the average of 200 simulations. Line-circles indicate the position of example simulations (A and C) and represent a small fraction of the total number of simulations shown in the plots (B and D).

in an improved quality of fit as highlighted by a reduction in the variability of the estimated output of tracer off-rate values. The results of Monte Carlo simulations using the kinetic association model equations are summarized in Supplemental Table 1. It must be emphasized that although our analysis focuses on the kinetic parameter $k_{\text {off }}$, the variability of $k_{\text {on }}$ of both tracer and competitor is also documented in Supplemental Tables 1-5.

\section{Competition Kinetic Binding between Tracer and Unlabeled Competitor Mimicking an Off-Line Addition Protocol}

To accurately determine the kinetics of unlabeled competitor compounds, it is conventional to construct a family of association kinetic curves using a fixed tracer concentration $\left(\sim 3 \times K_{\mathrm{d}}\right)$ and varying concentrations of the unlabeled compound with each association curve being monitored until equilibrium (see Fig. 2). In the case of the most slowly dissociating tracer, association curves were monitored for 180 minutes to reflect practical limitations. The resulting data were then fitted to the Motulsky-Mahan model, which describes the interaction between an unlabeled compound and a labeled tracer and allows us to calculate the $k_{\text {on }}$ and $k_{\text {off }}$ of the unlabeled compound (eq. 2).

Example results of Monte Carlo simulations using the Motulsky-Mahan model equation are shown in Fig. 2. In each case, the very rapidly dissociating tracer was employed; with input values of $k_{\text {on }}$ for the tracer fixed at $3 \times 10^{7} \mathrm{M}^{-1}$ minute ${ }^{-1}$ and $k_{\text {off }}$ fixed at 10 minute ${ }^{-1}$. Figure 2 differs only in respect of the kinetic properties of the unlabeled compound in competition with the tracer, with initial start times fixed at 1 second and with read frequency fixed every 10 seconds thereafter. Under these simulation conditions and in the presence of a rapidly dissociating unlabeled compound (with kinetic parameters; $k_{\text {off }}$ of 10 minute ${ }^{-1}, k_{\text {on }}$ of $1 \times 10^{6} \mathrm{M}^{-1}$ minute $^{-1}$ ), equilibrium between the rapidly dissociating tracer, competitor compound, and receptor is reached rapidly within the first 30 seconds (see Fig. 2A).

In direct contrast in the presence of a more slowly dissociating competitor (with kinetic parameters; $k_{\text {off }}$ of 1 minute $^{-1}, k_{\text {on }}$ of $1 \times 10^{7} \mathrm{M}^{-1}$ minute $^{-1}$ ) then the time to equilibrium is markedly increased with a characteristic "overshoot" in the initial binding of the tracer (see Fig. 2B). The data contained in Fig. 2 represent a single simulation 

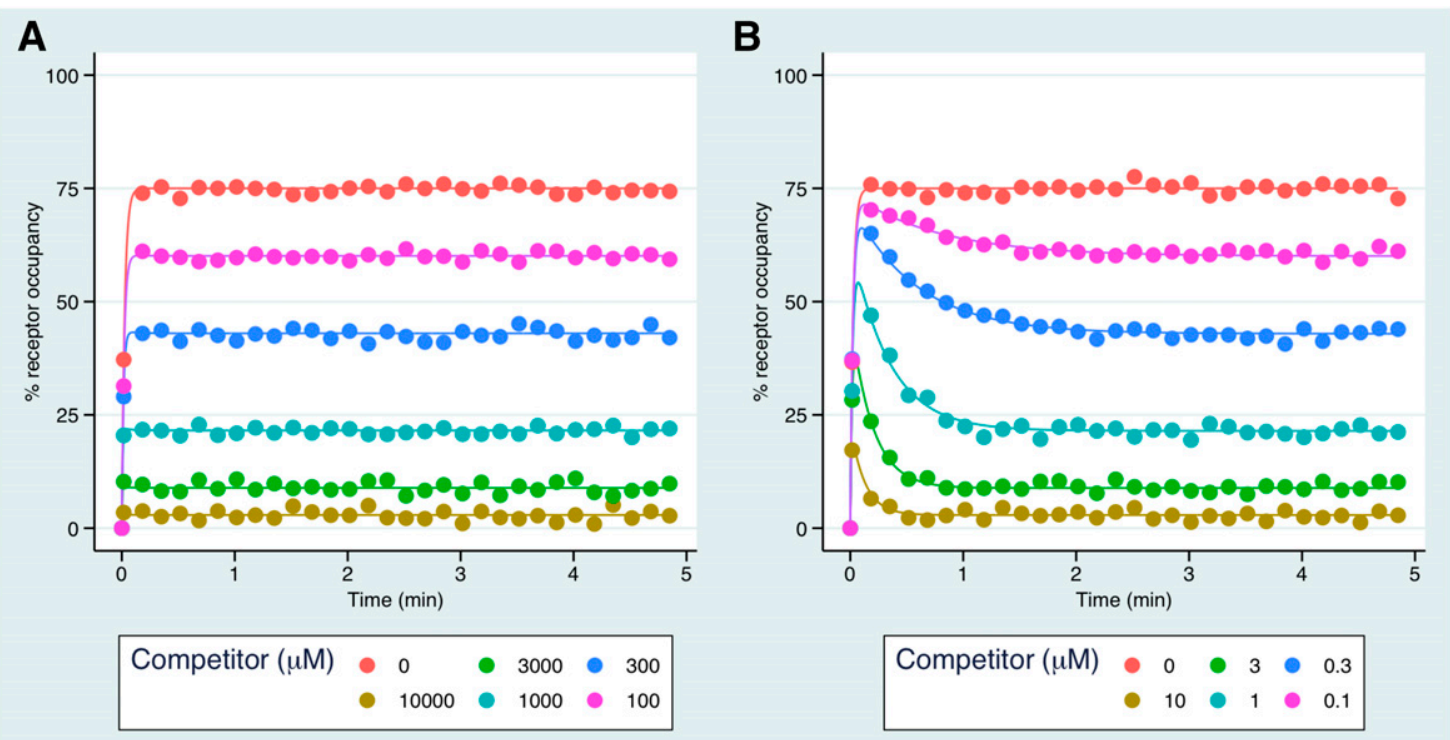

Fig. 2. Monte Carlo simulation results exploring the effect of competitor off-rate on the competition profile observed with a rapidly dissociating tracer. (A) Competition between a fixed concentration $\left(3 \times K_{\mathrm{d}}\right.$ ) of a very rapidly dissociating tracer; $k_{\text {off }}$ of 10 minute ${ }^{-1}, k_{\text {on }}$ of $3 \times 10^{7} \mathrm{M}^{-1} \mathrm{minute}^{-1}$ and a rapidly dissociating competitor with the following kinetic parameters; $k_{\text {off }}$ of 10 minute ${ }^{-1}, k_{\text {on }}$ of $1 \times 10^{6} \mathrm{M}^{-1} \cdot \mathrm{minute}^{-1}$, data shown are representative of 200 simulations. (B) Competition between a fixed concentration $\left(3 \times K_{\mathrm{d}}\right)$ of a very rapidly dissociating tracer and a slowly dissociating competitor with the following kinetic parameters; $k_{\text {off }}$ of 1 minute ${ }^{-1}, k_{\text {on }}$ of $1 \times 10^{7} \mathrm{M}^{-1} \cdot$ minute ${ }^{-1}$, data shown are representative of 200 simulations. In all cases, tracer and competitor binding simulations were performed with an initial start time of 1 second (representative of injection of receptor to a reaction containing tracer) and a read frequency of 10 seconds.

and is illustrative of the whole dataset, which is summarized in Fig. 3.

State of the art radioligand-based competition association binding assays are routinely formulated using only 12 time points (Sykes et al., 2010) employing either a single concentration of competitor (Sykes et al., 2014; Martella et al., 2017) or up to three concentrations of competitor (Sykes et al., 2009, 2010 ) and a tracer concentration in the range of $1-10 \times$ its own $K_{\mathrm{d}}$. Current knowledge of FRET-based competition association binding assays is based on a small number of studies, which in general have employed an off-line addition protocol to improve experimental throughput but also to allow greater temperature control during the initial mixing step (KleinHerenbrink et al., 2016; Sykes et al., 2017; Sykes and Charlton, 2018; Sykes et al., 2018).

Figure 3 shows summary off-rate data from the MotulskyMahan fits to the Monte Carlo kinetic simulations exploring the effect of sample time and tracer kinetics on our ability to determine accurately competitor kinetic parameters $\left(k_{\text {off }}\right.$ range from 100 to 0.01 minute $^{-1}$ ) and is representative of an off-line addition protocol with a 30 -second start time. In all cases, tracers were employed at a concentration $3 \times$ their own $K_{\mathrm{d}}$ value. These results demonstrate that even the very rapidly dissociating tracer examined $\left(k_{\text {off }}=10\right.$ minute $\left.^{-1}\right)$ is not able to accurately determine the kinetics of unlabeled compounds with off-rates ranging from 100 to 10 minute $^{-1}$ (see Fig. 3A). This represents a limitation in the kinetic quantification of unlabeled compounds using the competition association method where reagents are added offline from the plate reader.

Also apparent was the influence of read frequency on our ability to accurately predict the kinetics of unlabeled competitor compounds with slower dissociation rates, i.e., those in the range of 0.01 minute $^{-1}$, reflecting the imprecise fitting of the tracer "overshoot." Interestingly, a tracer with a $k_{\text {off }}$ of 1 min$u \mathrm{ut}^{-1}$ under the conditions explored could not accurately fit the kinetics of unlabeled compounds with a $k_{\text {off }}$ of 100 minute $^{-1}$ and is only suitable to unambiguously and accurately quantify the kinetics of unlabeled compounds with a $k_{\text {off }}$ in the range of 10 minute $^{-1}$ if the read frequency remains at 1 second (see Fig. 3B). Almost identical patterns were observed for tracers with $k_{\text {off }}$ values of 0.1 and 0.01 minute $^{-1}$ (see Fig. 3, $\mathrm{C}$ and D). The results of Monte Carlo simulations using the Motulsky-Mahan model equation are summarized in Supplemental Tables 2-5.

\section{Competition Kinetic Binding between Tracer and Unlabeled Competitor Mimicking an On-Line Injection Protocol}

Having established the limitation of the off-line injection protocol, we decided to explore the potential of utilizing onboard plate reader injectors and the increased resolution at very early time points to improve the goodness of fit (fit quality). Figure 4 shows summary dissociation rate estimates obtained by fitting Monte Carlo simulations that explore the effect of read frequency and tracer kinetics on our ability to accurately determine competitor kinetic parameters $\left(k_{\text {off }}\right.$ range from 100 to 0.01 minute $^{-1}$ ) and is representative of an injection protocol with a 1-second start time. In all cases, tracers were employed at a concentration $3 \times$ their own $K_{\mathrm{d}}$ value. These simulations demonstrate that the very rapidly dissociating tracer examined $\left(k_{\text {off }}=10\right.$ minute $\left.^{-1}\right)$ is able to accurately determine the kinetics of unlabeled compounds with off-rates ranging from 100 to 0.01 minute $^{-1}$ (see Fig. 4A). Interestingly under these tracer conditions employed, the range of dissociation rate estimates markedly increases for the most rapidly dissociating unlabeled compounds examined (10-100 minute $\left.^{-1}\right)$, representing the limit of kinetic detection 


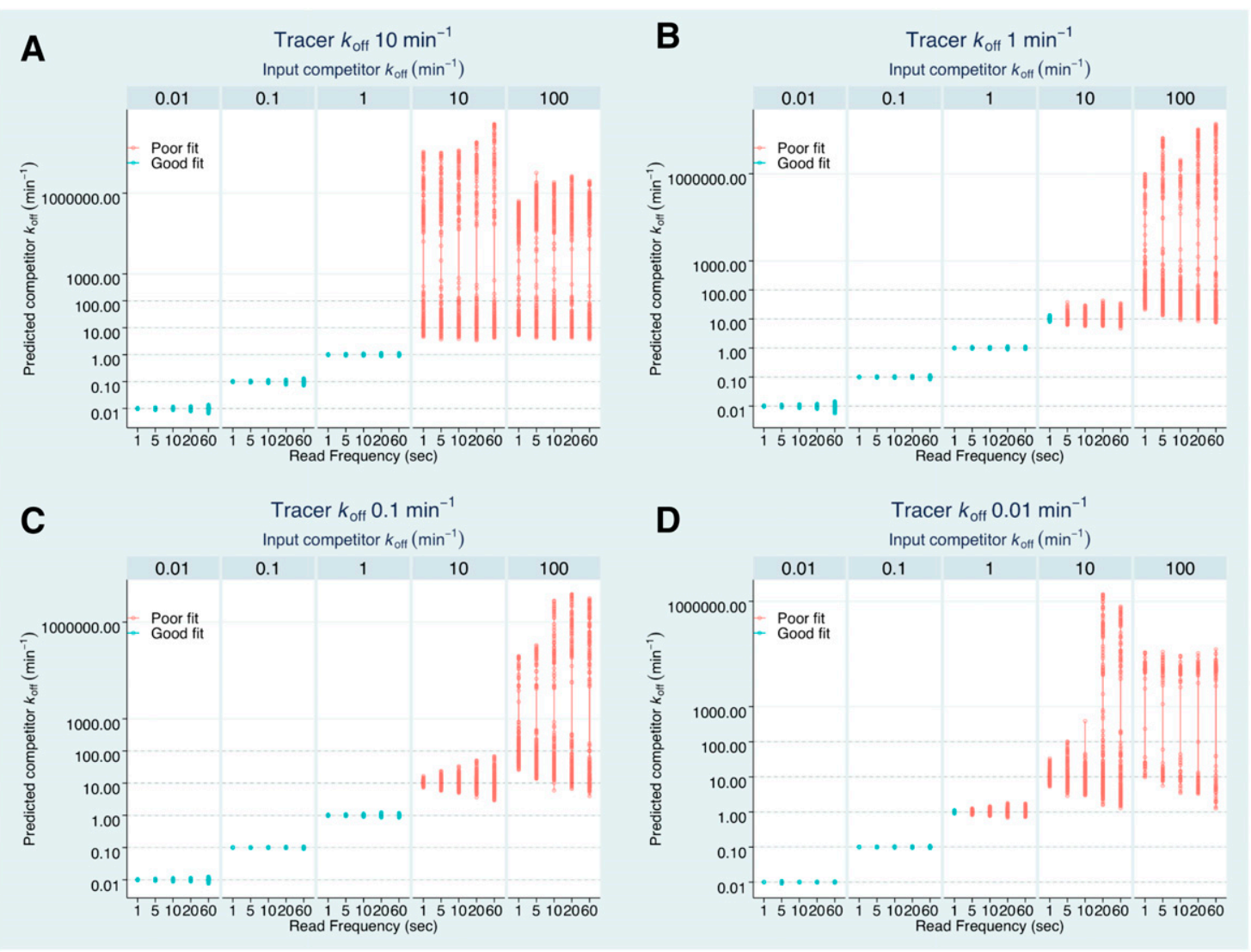

Fig. 3. Monte Carlo simulation results exploring the effect of sample time and tracer kinetics on the accurate determination of competitor $k_{\text {off }}$ representative of an off-line addition protocol. Individual panels show the effect of assay read frequency time on the measured $k_{\text {off }}$ of unlabeled competitor compounds with varied kinetics in competition with a fixed concentration $\left(3 \times K_{\mathrm{d}}\right)$ of a very rapidly dissociating tracer with the following kinetic parameters, $k_{\text {off }}$ of 10 minute ${ }^{-1}, k_{\text {on }}$ of $3 \times 10^{7} \mathrm{M}^{-1}$ minute $^{-1}(\mathrm{~A})$; a rapidly dissociating tracer with the following kinetic parameters, $k_{\text {off }}$ of 1 minute ${ }^{-1}, k_{\text {on }}$ of $1 \times 10^{7} \mathrm{M}^{-1}$ minute $^{-1}$ (B); a slowly dissociating tracer with kinetic parameters, $k_{\text {off }}$ of 0.1 minute ${ }^{-1}, k_{\text {on }}$ of $1 \times 10^{8} \mathrm{M}^{-1}$ minute mine $^{-1}$ (C); and a very slowly dissociating tracer with kinetic parameters, $k_{\text {off }}$ of 0.01 minute $^{-1}, k_{\text {on }}$ of $1 \times 10^{9} \mathrm{M}^{-1}$ minute ${ }^{-1}(\mathrm{D})$. Blue symbols represent conditions that returned $>90 \%$ reliable fits. Red symbols represent conditions that returned $<90 \%$ reliable fits. In all cases tracer simulations were performed with an initial start time of 30 seconds representative of off-line addition of receptor to a reaction containing free tracer and unlabeled competitor, absolute values are the average of 200 simulations.

for this type of competition kinetic binding approach (see Supplemental Table 2). Also apparent was the influence of increasing read frequency on our ability to accurately predict the kinetics of unlabeled compounds with slower dissociation rates in the range of 0.01 minute $^{-1}$. Increased variation likely reflects the imprecise fitting of the tracer "overshoot," which is reliant on early time points for accuracy. In contrast, a tracer with a $k_{\text {off of }} 1$ minute $^{-1}$ is seemingly suitable for the determination of off-rates in the range of $\sim 10$ minute $^{-1}$, but increasing variation in the mean is observed as the read frequency is increased from 1 to 60 seconds (see Fig. 4B). Practically this wider degree of variation in the mean will become especially apparent if the number of observations for a particular compound is kept low (i.e., $n \leq 4$ ). A different pattern is observed for a tracer with a $k_{\text {off }}$ value equal to 0.1 minute $^{-1}$, as shown in Fig. 4C, for compounds with rapid offrates between 10 and 100 minute $^{-1}$ the number of ambiguous fits is very high (see Supplemental Table 4), and it is only compounds with $k_{\text {off }}$ value of $\leq 1$ minute $^{-1}$ that can be measured without ambiguity at this concentration of tracer employed (i.e., $3 \times K_{\mathrm{d}}$ ). Interestingly the appearance of ambiguity in fitting estimates precedes any inaccuracies in the fitting estimates themselves. Finally a kinetic tracer with a $k_{\text {off }}$ of 0.01 minute $^{-1}$ employed at a concentration of $3 \times K_{\mathrm{d}}$ is only able to determine unambiguously (and accurately) the kinetics of compounds with $k_{\text {off }} \leq 1$ minute $^{-1}$ when the read frequency is shortened to 1 second (see Fig. 4D) and as a consequence is of limited value as a tracer for kinetic determinations of more rapidly dissociating compounds.

The figures that are derived from these Monte Carlo simulations are useful guides to enable investigators interested in compound kinetics and associated structure activity relationships to set up kinetic screens and choose appropriate tracers to profile the properties of unlabeled compounds. To illustrate some key points, Monte Carlo simulation results reproducing the experimentally observed effect of competitor off-rate on the competition profile observed with a rapidly dissociating tracer and a slowly dissociating tracer with varying start time are depicted in Fig. 5. Figure 5A shows competition between a fixed concentration $\left(3 \times K_{\mathrm{d}}\right)$ of a very slowly dissociating tracer $\left(k_{\text {off }}\right.$ of 0.01 minute $^{-1}, k_{\text {on }}$ of $1 \times 10^{9} \mathrm{M}^{-1}$ minute ${ }^{-1}$ ) and unlabeled competitors with varying dissociation rates. What becomes apparent is that despite the variation in the unlabeled compound off-rate $\left(0.01-100\right.$ minute $\left.^{-1}\right)$, the tracer association curves in the presence of the compounds with a $k_{\text {off }}>0.01$ become bunched to the point they become practically indistinguishable. Figure $5 \mathrm{C}$ was obtained under identical conditions apart from a 30 -second delay in 

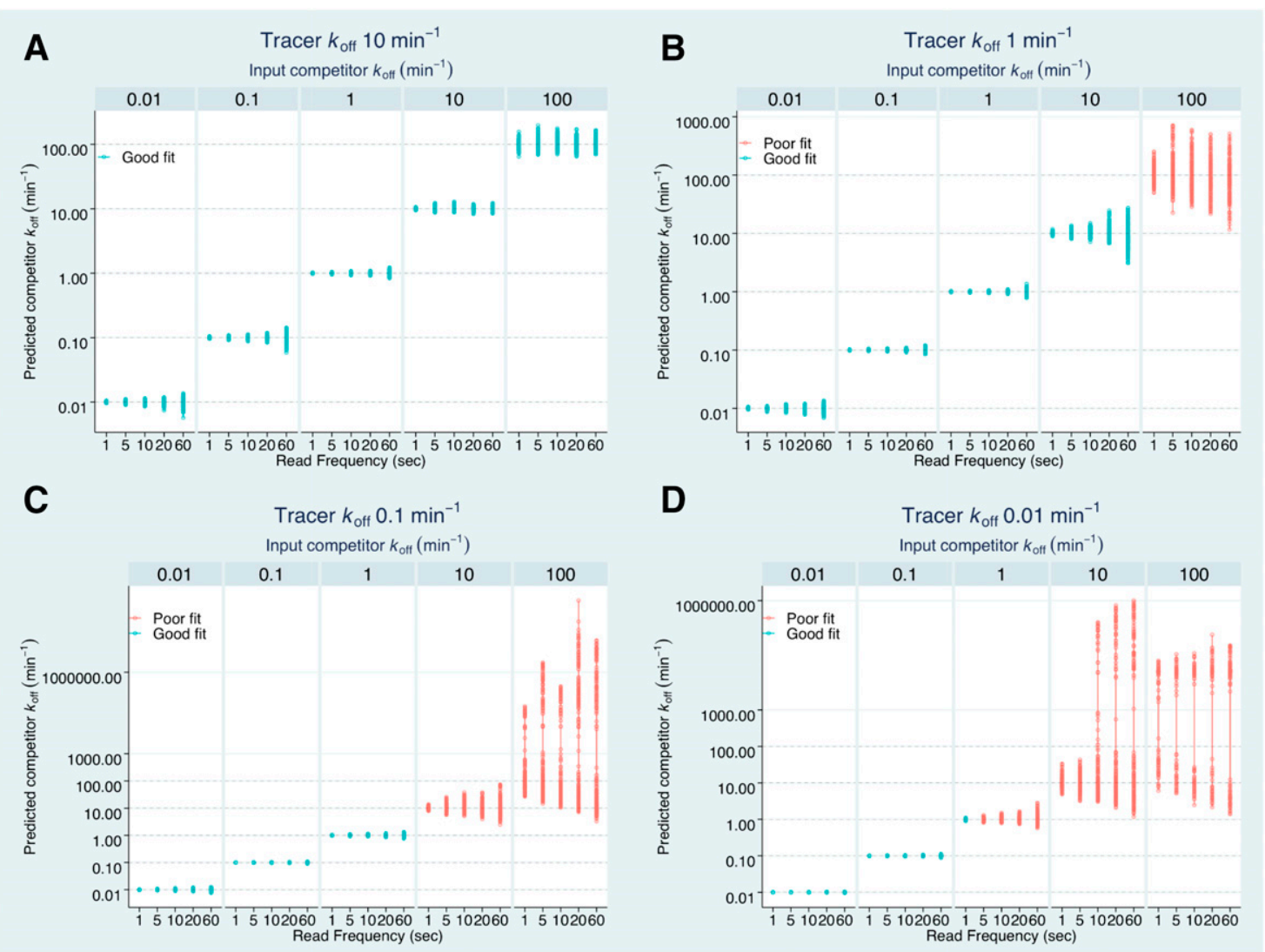

Fig. 4. Monte Carlo simulation results exploring the effect of tracer kinetics and sample time on the accurate determination of competitor $k_{\text {off }}$ representative of an injection protocol. Individual figures show the effect of assay read frequency on the measured $k_{\text {off }}$ of unlabeled competitor compounds with varied kinetics in competition with a fixed concentration $\left(3 \times K_{\mathrm{d}}\right)$ of a very rapidly dissociating tracer with the following kinetic parameters, $k_{\text {off }}$ of 10 minute ${ }^{-1}, k_{\text {on }}$ of $3 \times 10^{7} \mathrm{M}^{-1} \cdot$ minute $^{-1}(\mathrm{~A})$; a rapidly dissociating tracer with kinetic parameters, $k_{\text {off }}$ of 1 minute ${ }^{-1}, k_{\text {on }}$ of $1 \times 10^{7} \mathrm{M}^{-1} \cdot \mathrm{minute}^{-1}(\mathrm{~B})$; a slowly dissociating tracer with kinetic parameters, $k_{\text {off }}$ of 0.1 minute ${ }^{-1}, k_{\text {on }}$ of $1 \times 10^{8} \mathrm{M}^{-1}$. minute ${ }^{-1}(\mathrm{C})$; and a very slowly dissociating tracer with kinetic parameters $k_{\text {off }}$ of 0.01 minute ${ }^{-1}, k_{\text {on }}$ of $1 \times 10^{9} \mathrm{M}^{-1}$. minute ${ }^{-1}(\mathrm{D})$. Blue symbols represent conditions that returned $>90 \%$ reliable fits. Red symbols represent conditions that returned $<90 \%$ reliable fits. In all cases tracer simulations were performed with an initial start time of 1 second representative of injection of receptor to a reaction containing free tracer and unlabeled competitor; absolute values are the average of 200 simulations. $k_{\text {obs }} t_{1 / 2}$ values $\left(0.693 / k_{\text {obs }}\right)$ for the tracers with dissociation rates of $10,1,0.1$, and 0.01 minute $^{-1}$ were $1.1,10.4$ seconds and 1.7 and 17.3 minutes, respectively.

start time, representative of off-line addition. The increased start time has little effect on the ability to discriminate between the kinetic off-rates of these compounds primarily as the tracer itself is slow to reach equilibrium with the receptor.

Figure 5B shows competition between a fixed concentration $\left(3 \times K_{\mathrm{d}}\right)$ of a rapidly dissociating tracer $\left(k_{\text {off }}\right.$ of 10 minute $^{-1}, k_{\text {on }}$ of $3 \times 10^{7} \mathrm{M}^{-1}$ minute $^{-1}$ ) and unlabeled competitors with varying dissociation rates $\left(0.01-100\right.$ minute $\left.^{-1}\right)$ and demonstrates how it is possible to accurately determine the dissociation rates of the more rapidly dissociating competitors using the injection protocol $\left(k_{\text {off }}\right.$ range $0.01-10$ minute $^{-1}$, assay start time 1 second). Figure 5D was obtained under identical conditions apart from a 30-second delay in start time representative of off-line addition. This time the increased start time has a major effect on the ability of this tracer to discriminate between the kinetic off-rates of these compounds primarily as the tracer itself very rapidly reaches equilibrium with the receptor. Interestingly, this loss of early time points in the case of an unlabeled competitor with a $k_{\text {off }}$ of 10 minute $^{-1}$ results in an apparent underestimation of the $k_{\text {off }}$ of the unlabeled competitor as demonstrated by the apparent tracer "overshoot." This highlights the key importance of the early time points for accurate competitor characterization.
In line with previous kinetic studies (Klein-Herenbrink et al., 2016), the $k_{\text {off }}$ of unlabeled compounds was poorly estimated when the tracer $k_{\text {off }}$ is substantially slower than that of the unlabeled ligand. Increasing the $k_{\text {off }}$ of the tracer from 0.1 to 10 vastly improved the precision with which the values of $k_{\text {on }}$ and $k_{\text {off }}$ were estimated for the more rapidly dissociating compounds (as illustrated in Supplemental Tables 2-5). In contrast, $k_{\text {on }}$ had no impact on the accuracy of the parameter estimate reflective of the fact that changes in $k_{\text {on }}$ are completely compensated for by changes in the ligand concentration (data not shown), i.e., no change in $k_{\text {obs }}$.

\section{Effect of Tracer Concentration on Kinetic Determination}

The effect of tracer concentration on the accuracy of kinetic determinations was explored in a further series of Monte Carlo simulations. Tracer concentration was both lowered to $1 \times K_{\mathrm{d}}$ and increased to 10 and $30 \times K_{\mathrm{d}}$. We chose to highlight a tracer with kinetic properties commonly encountered in drug-receptor screening campaigns: high affinity $(1 \mathrm{nM})$, fast on $1 \times 10^{8} \mathrm{M}^{-1}$ minute $^{-1}$, and slow off 0.1 minute $^{-1}$. The results obtained with this tracer in competition with compounds with kinetic off rates ranging from 100 to 0.01 minute $^{-1}$ following an on-line addition protocol are shown in Fig. 6 . 

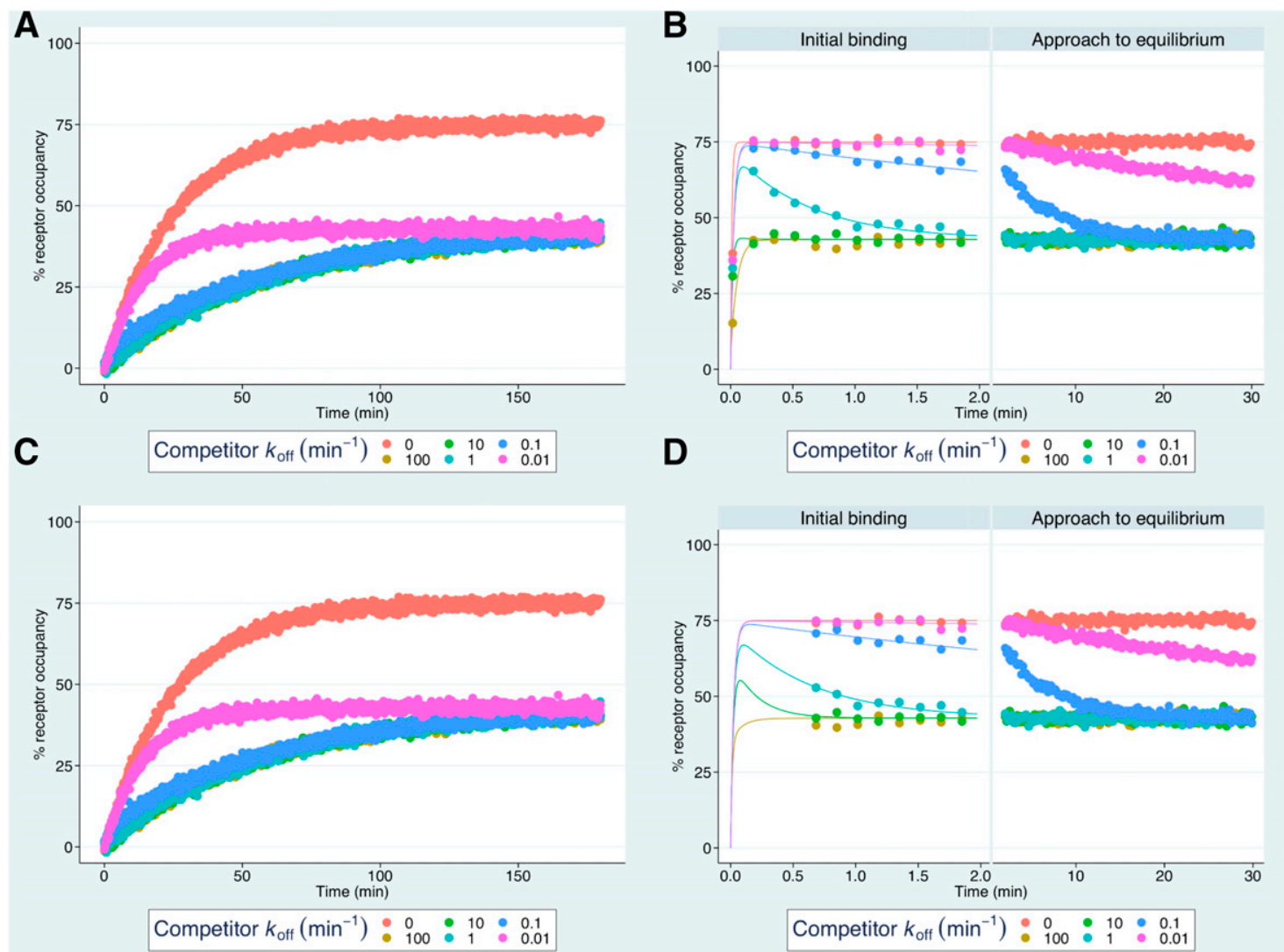

Fig. 5. Monte Carlo simulation results reproducing the experimentally observed effect of competitor off-rate on the competition profile observed with a rapidly dissociating tracer and a slowly dissociating tracer at varying start times. (A and C) show competition between a fixed concentration $\left(3 \times K_{\mathrm{d}}\right)$ of a very slowly dissociating tracer with the following kinetic parameters: $k_{\text {off }}$ of 0.01 minute ${ }^{-1}, k_{\text {on }}$ of $1 \times 10^{9} \mathrm{M}^{-1} \cdot$ minute $^{-1}$ and competitors with varying dissociation-rates. (B and D) show competition between a fixed concentration $\left(3 \times K_{\mathrm{d}}\right)$ of a rapidly dissociating tracer with the following kinetic parameters: $k_{\text {off of }} 10$ minute ${ }^{-1}, k_{\text {on }}$ of $3 \times 10^{7} \mathrm{M}^{-1}$.minute ${ }^{-1}$ and competitors with varying dissociation rates. In the case of (A and B), tracer and competitor binding simulations were performed with an initial start time of 1 second (representative of on-line injection of receptor to a reaction containing tracer and competitor), whereas (C and D) were performed with a start time of 30 seconds (representative of off-line addition of receptor). All simulations have a read frequency of 10 seconds, and the data shown are representative of 200 simulations.

Simulations varying concentration following an on-line addition protocol were also performed for tracers with offrates of 10, 1, and 0.01 as shown in Supplemental Figs. 1-3. $k_{\text {obs }} t_{1 / 2}$ values $\left(0.693 / k_{\text {obs }}\right)$ for the tracers with dissociation rates of $10,1,0.1$, and 0.01 minute $^{-1}$ were $1.1,10.4$ seconds, 1.7 , and 17.3 minutes, respectively, at tracer concentrations $3 \times$ their respective $K_{\mathrm{d}}$.

As a generality, an increase in ligand concentration was associated with an improvement in both the accuracy of kinetic determinations but also the number of fits deemed unambiguous and, in some cases, effectively rescued the ability of a tracer to determine the kinetics of a more rapidly dissociating competitor. For example, a competitor with an offrate of 10 minute $^{-1}$ competing with a tracer with an off rate of 0.1 minute $^{-1}$ could be readily resolved when the tracer concentration was increased from $3 \times K_{\mathrm{d}}$ (Fig. $6 \mathrm{~B}$ ) to $30 \times K_{\mathrm{d}}$ (Fig. 6D). $k_{\text {obs }} t_{1 / 2}$ values for a tracer with a dissociation rate of 0.1 minute $^{-1}$ ranged from 3.5 to 0.2 minutes at concentrations of tracer $1-30 \times K_{\mathrm{d}}$, respectively. Data for a competitor with an off-rate of 10 minute $^{-1}$ competing with different concentrations of tracers with off-rates ranging from 0.01 to 10 minute $^{-1}$ are summarized in Fig. 7.

Similarly the effect of tracer concentration was explored following an off-line addition protocol with the data summarized in Supplemental Figs. 4-7. In the case of a tracer with an off-rate of 0.1 minute $^{-1}$, increasing tracer concentration with the offline method did not improve the goodness of fit when quantifying competitors with off-rates in the region of 10 minute $^{-1}$, but tuning this particular experimental variable may still offer some benefit to researchers without access to detection instruments with injectors, reducing the overall error associated with kinetic determinations.

\section{Fragment Screening Using Competition-Kinetic Association Binding Assays}

Monte Carlo simulations were performed to recreate the competition profile expected with low-affinity fragments (31 in total with $K_{\mathrm{d}}$ values ranging from 1 to $\left.1000 \mu \mathrm{M}\right)$. These competitor fragments with varied kinetic parameters $\left(k_{\text {off }}\right.$ ranging from 3 to 200 minute $^{-1}$ and $k_{\text {on }}$ from $3 \times 10^{6}$ to $2 \times 10^{5}$ $\mathrm{M}^{-1}$ minute $^{-1}$ ) were simulated in competition with a fixed concentration $\left(3 \times K_{\mathrm{d}}\right)$ of a rapidly dissociating tracer $\left(k_{\text {off }}\right.$ of 10 minute $^{-1}, k_{\text {on }}$ of $3 \times 10^{7} \mathrm{M}^{-1}$ minute $\left.^{-1}\right)$. Supplemental Fig. $8 \mathrm{~A}$ shows the expected inhibition of tracer binding caused by a low-affinity competitor, fragment 25 (affinity of $0.25 \mathrm{mM}, k_{\text {off }}$ of 87.1 minute $^{-1}, k_{\text {on }}$ of $5 \times 10^{5} \mathrm{M}^{-1}$ minute $^{-} 1$ ) tested at two concentrations, 10 and $100 \mu \mathrm{M}$. Using a start time of 1 second and a read frequency of 5 seconds, we were able to estimate the affinity of such fragments with a high degree of accuracy as 

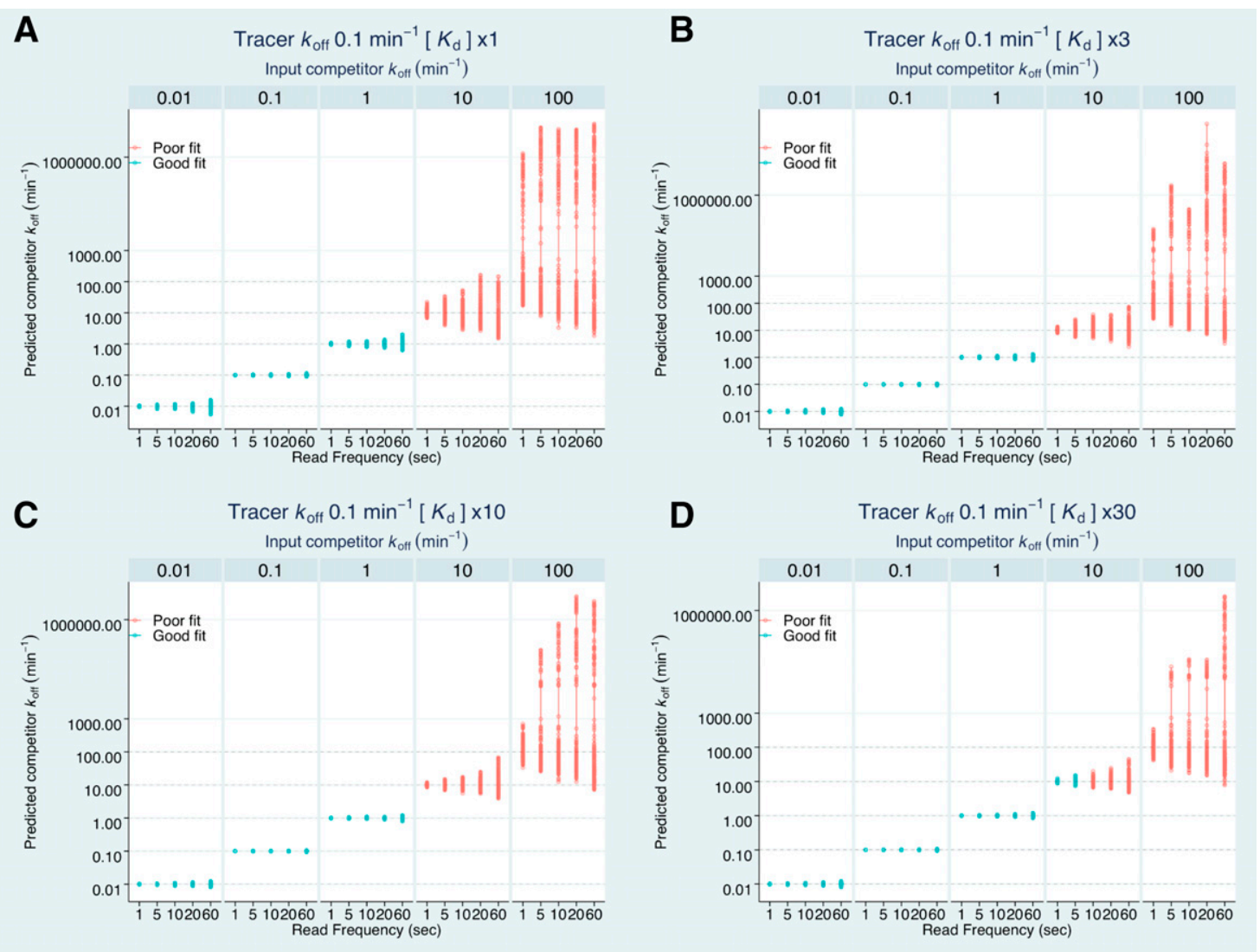

Fig. 6. Monte Carlo simulation results exploring the effect of tracer concentration and sample time on the accurate determination of competitor $k_{\text {off }}$ representative of an injection protocol. Effect of assay read frequency on measured $k_{\text {off }}$ of unlabeled competitor compounds with varied kinetics in competition with different concentrations of a slowly dissociating tracer with kinetic parameters, $k_{\text {off }}$ of 0.1 minute ${ }^{-1}, k_{\text {on }}$ of $1 \times 10^{8} \mathrm{M}^{-1} \cdot \mathrm{minute}^{-1}$. Tracer concentrations were $1 \times K_{\mathrm{d}}(\mathrm{A}), 3 \times K_{\mathrm{d}}(\mathrm{B}), 10 \times K_{\mathrm{d}}(\mathrm{C})$, and $30 \times K_{\mathrm{d}}(\mathrm{D})$. Blue symbols represent conditions that returned $>90 \%$ reliable fits. Red symbols represent conditions that $<90 \%$ reliable fits. In all cases tracer simulations were performed with an initial start time of 1 second representative of injection of receptor to a reaction containing free tracer (L) and unlabeled competitor (I), absolute values are the average of 200 simulations. $k_{\text {obs }} t_{1 / 2}$ values $\left(0.693 / k_{\mathrm{obs}}\right)$ for a tracer with a dissociation rate of 0.1 minute ${ }^{-1}$, with increasing concentration of tracer $1,3,10$ and $30 \times K_{\mathrm{d}}$ were $3.5,1.7,0.6$ and 0.2 minute respectively.

shown by the correlation of input $K_{\mathrm{d}}$ with output $K_{\mathrm{d}}$ (see Supplemental Fig. 8B). In contrast, as the $k_{\text {off }}$ of the fragment increases then the degree of error associated with the estimation of $k_{\text {off }}$ and $k_{\text {on }}$ is increased (as judged by the increase in the overall S.D. associated with the mean); however, the overall correlation of input and output kinetic parameters remains extremely good (see Supplemental Fig. 8, C and D). In all cases, using this two-point method the number of ambiguous fits was below $5 \%$. Interestingly this analysis demonstrates that the affinity of the fragments is estimated with a much higher degree of accuracy than the individual kinetic measurements, demonstrating the overriding influence of the final equilibrium position on kinetic parameter estimation. A similar analysis was performed using single concentrations of compound (10 or $100 \mu \mathrm{M})$ and clearly demonstrates the benefit of the twopoint approach (see Turner and Charlton, 2005), with the errors associated, with each estimate at single concentrations being larger, and the number of ambiguous fits increasing proportionally (see Supplemental Fig. 9, A-F). Details on how to perform a Monte Carlo analysis and an example Monte Carlo Prism file can be found in the Supplemental methods (see "How to conduct a Monte Carlo Analysis in GraphPad Prism").

\section{Experimental Validation of the Monte Carlo Simulation Approach}

To validate the findings of these Monte Carlo simulations exploring the effect of tracer characteristics on kinetic determinations using the Motulsky-Mahan approach, we undertook an experimental study designed to explore the limits of a previously well characterized system, the dopamine D2L receptor using a TR-FRET-based system. In this study we chose to focus on a small number of compounds with diverse kinetics and employ the BMG pherastar FS (plate reader) injectors to make an assessment of tracer binding at the very earliest time points.

\section{Determining the Kinetic of $D_{\mathbf{2}} R$ Fluorescent Tracers}

Representative kinetic association curves for PPHT-red and spiperone-d2 using a start time of $\sim 1$ second and a read frequency of 5 seconds are shown in Fig. 8, A and C, respectively. The kinetic parameters determined from these plots are presented in Table 2. PPHT-red dissociated rapidly from the dopamine $\mathrm{D}_{2} \mathrm{R}$ with a $t_{1 / 2}$ of 2.2 minutes $\left(0.693 / k_{\text {off }}\right)$, whereas spiperone-d2 dissociated more slowly $\left(t_{1 / 2}\right.$ of 8.7 minutes). The removal of data points to reflect off-line addition (i.e., the first $\sim 30$ seconds) or increasing read frequencies 

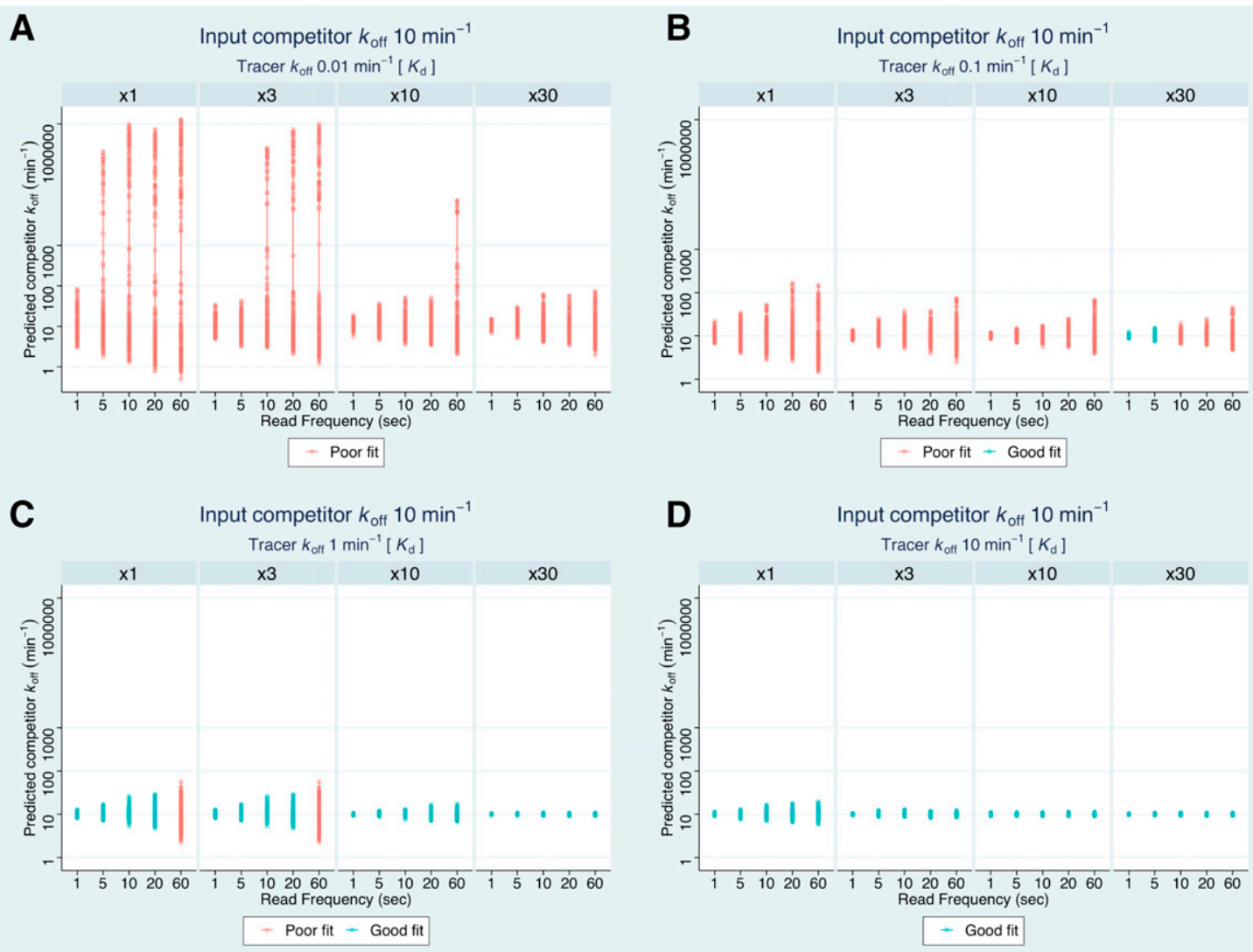

Fig. 7. Monte Carlo simulation results exploring the effect of tracer kinetics, concentration and sample time on the accurate determination of $k_{\text {off }}$ of a rapidly dissociating competitor representative of an on-line addition protocol. Effect of tracer concentration $\left(1-30 * K_{\mathrm{d}}\right)$ and read frequency $(1-60$ seconds) on the measured $k_{\text {off }}$ of an unlabeled competitor compound with a $k_{\text {off }}$ of 10 minute ${ }^{-1}$ in competition with different concentrations of a tracers with varied kinetic $k_{\text {off }}$ rates ranging from 0.01 to 10 minute $^{-1}$. Tracer off-rates were 0.01 (A), 0.1 (B), 1 (C), and 10 minute ${ }^{-1}$ (D). Blue symbols represent conditions that returned $>90 \%$ reliable fits. Red symbols represent conditions that returned $<90 \%$ reliable fits. In all cases, tracer simulations were performed with an initial start time of 1 second representative of addition of receptor to a reaction containing free tracer and unlabeled competitor, absolute values are the average of 200 simulations.

(10-60 seconds) had little influence on the determination of the kinetic parameters of these two fluorescent tracers reflective of the fact that they are not so rapidly dissociating that the early time points are critical for an accurate estimation of their kinetic parameters. Figure 8, B and D, show the expected linear relationship between PPHT-red and spiperone-d2 concentration and the observed rate of association (or $k_{\mathrm{obs}}$ ) and suggest that the Laws of Mass Action are observed.

\section{Effect of Tracer Kinetics on Dopamine $\mathrm{D}_{2} \mathrm{R}$ Ligand Kinetic Determinations}

To experimentally test the effect of tracer kinetics on $k_{\text {off }}$ estimates of unlabeled ligands, competition association experiments were performed for five dopamine D2 ligands: chlorpromazine, ropinerole, pergolide, domperidone, and butaclamol (Fig. 9). The associated errors and therefore the accuracy of kinetic determinations for rapidly dissociating compounds is highly dependent on the kinetic properties of the tracer employed to measure them. In contrast, the error associated with more slowly dissociating compounds is largely comparable and seemingly independent of the tracer's kinetic properties. This is illustrated in Fig. 9 and shows that the error associated with kinetic determinations made with the more slowly dissociating tracer spiperone- $\mathrm{d} 2$ is in general much larger than for the more rapidly dissociating tracer PPHT-red. This is evident from the spread of $k_{\text {off }}$ values on the $x$-axis (spiperone-d2) being wider than the spread of the $k_{\text {off }}$ values on the $y$-axis (PPHT-red). The errors associated with kinetic determinations of rapidly dissociating compounds following off-line addition of PPHT-red (Fig. 9A) is little changed compared with on-line addition (Fig. 9B); however, there was a tendency for the error to increase for the more slowly dissociating compounds and this situation is replicated for spiperone-d2 (see Fig. 9) and potentially represents the imprecise fitting of the tracer "overshoot."

\section{Discussion}

The growing awareness of the importance of optimizing drug-binding kinetics has led to a rapid increase in the development and utilization of assay systems capable of measuring the kinetics of unlabeled compounds. A popular format for investigating membrane-bound targets is the competition association binding assay, first described by Arányi (1980) and then popularized by Motulsky and Mahan (1984) and used extensively to characterize many different receptor systems (Gillard et al., 2002; Dowling and Charlton, 2006; Sullivan et al., 2006; Fleck et al., 2012; Sykes et al., 2014; 
A

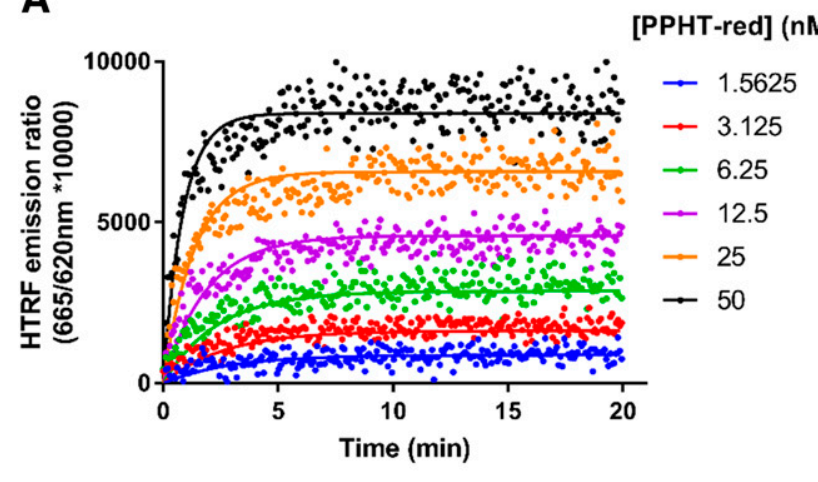

C

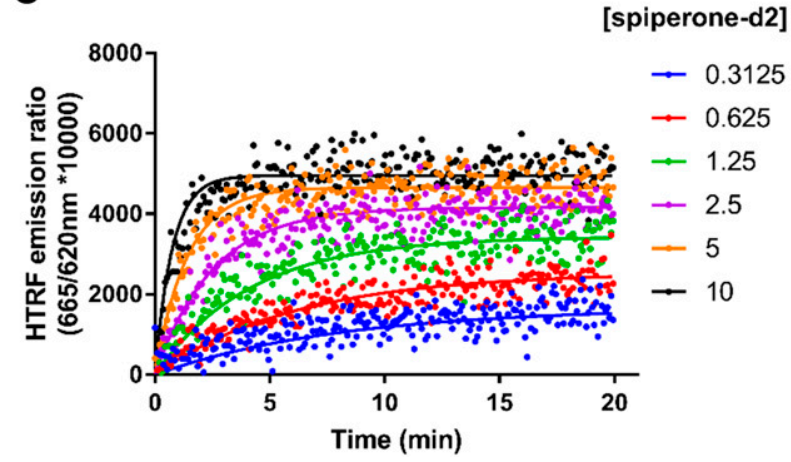

B

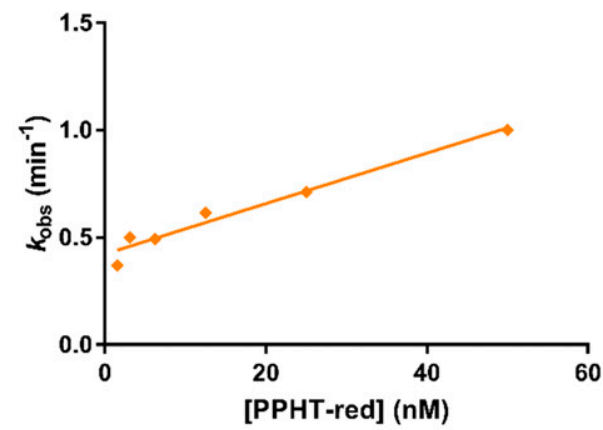

D

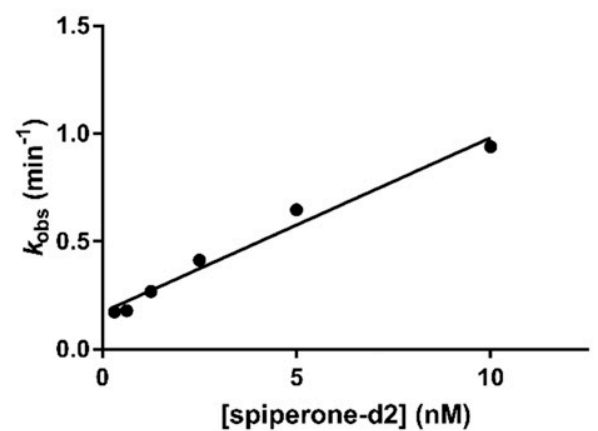

Fig. 8. Determination of PPHT-red and spiperone-d2 kinetic binding parameters. (A) Observed association of PPHT-red binding to the human dopamine $\mathrm{D}_{2} \mathrm{R}$. CHO- $\mathrm{D}_{2} \mathrm{R}$ cell membranes $(2 \mu \mathrm{g} / \mathrm{well}$ ) were incubated for 20 minutes with gentle agitation with increasing concentrations of PPHT-red. Data are presented in singlet form from a representative of four experiments. (B) Plot of PPHT-red concentration vs. $k_{\text {obs }}$. Binding followed a simple Law of Mass Action model, $k_{\mathrm{obs}}$ increasing in a linear manner with fluorescent ligand concentration. Data are representative of a total of four experiments. (C) Observed association of spiperone-d2 binding to the human dopamine $\mathrm{D}_{2} \mathrm{R}$. CHO- $\mathrm{D}_{2} \mathrm{R}$ cell membranes $(2 \mu \mathrm{g} /$ well $)$ were incubated for 20 minutes with gentle agitation with increasing concentrations of spiperone-d2. Data are presented in singlet form from a representative of four experiments. (D) Plot of spiperone-d 2 concentration vs. $k_{\text {obs. }}$. Binding followed a simple Law of Mass Action model, $k_{\text {obs }}$ increasing in a linear manner with fluorescent ligand concentration. Data are representative of a total of four experiments. All binding reactions were performed in the presence of $\mathrm{GppNHp}(0.1 \mathrm{mM}) \mathrm{with}$ nonspecific-binding levels determined by inclusion of haloperidol $(10 \mu \mathrm{M})$.

Riddy et al., 2015; Nederpelt et al., 2016; Guo et al., 2017; Bouzo-Lorenzo et al., 2019). A key observation during this time has been that the accuracy of estimating kinetic parameters of rapidly dissociating molecules is poorer when using a slowly dissociating ligand as the tracer (Sykes and Charlton, 2012; Klein-Herenbrink et al., 2016; Bosma et al., 2019).

The purpose of the current study was to explore the effect of tracer kinetics on our ability to accurately estimate the kinetics of unlabeled compounds using the competitionassociation binding method. In particular, we were interested to test the limits of this model system in terms of its ability to assess the binding of very rapidly dissociating compounds likely to be representative of compounds identified in a screening campaign. In practical terms, we were also keen to investigate the importance of read frequency to assist with experimental design. To our knowledge, the current study is the first to fully explore these elements and provide clear guidance for the use of this assay format at all stages of the drug discovery process.

As shown in Fig. 1 and Supplemental Table 1, it was generally possible to derive accurate estimates $(<2$-fold difference) of the tracer kinetic parameters using the global association eq. 1 for simulated datasets. For the more rapidly dissociating tracers, the key to obtaining more accurate kinetic parameters was to reduce the assay start time and the read frequency. Our ability to control these parameters is largely instrument (reader) dependent. For example, assay start time is dependent upon the ability to inject sample while simultaneously reading from the same well. In addition, assay sensitivity determines the required sample acquisition time, which restricts minimal read frequency. Consequently the option to vary these parameters can be considered as critical factors in the process of determining unlabeled compound kinetics. This is readily illustrated in a comparison of Fig. 1, B

TABLE 2

Kinetic binding parameters of the tracers PPHT-red and spiperoine-d2 determined from association binding studies using human dopamine $\mathrm{D}_{2 \mathrm{~L}} \mathrm{CHO}$ cell membranes Data are mean S.E.M. from $n$ separate experiments.

\begin{tabular}{lcccc}
\hline Tracer & Tracer $K_{\mathrm{D}}$ & Tracer $k_{\text {off }}$ & Tracer $k_{\text {on }}$ & No. of Observations \\
\hline & $n M$ & $\mathrm{~min}^{-1}$ & $M^{-1} \cdot \mathrm{min}^{-1}$ & \\
PPHT-red & $16.9 \pm 1.1$ & $0.32 \pm 0.02$ & $1.93 \pm 0.21 \times 10^{7}$ & 4 \\
Spiperone-d2 & $0.62 \pm 0.08$ & $0.08 \pm 0.01$ & $1.30 \pm 0.17 \times 10^{8}$ & 4 \\
\hline
\end{tabular}




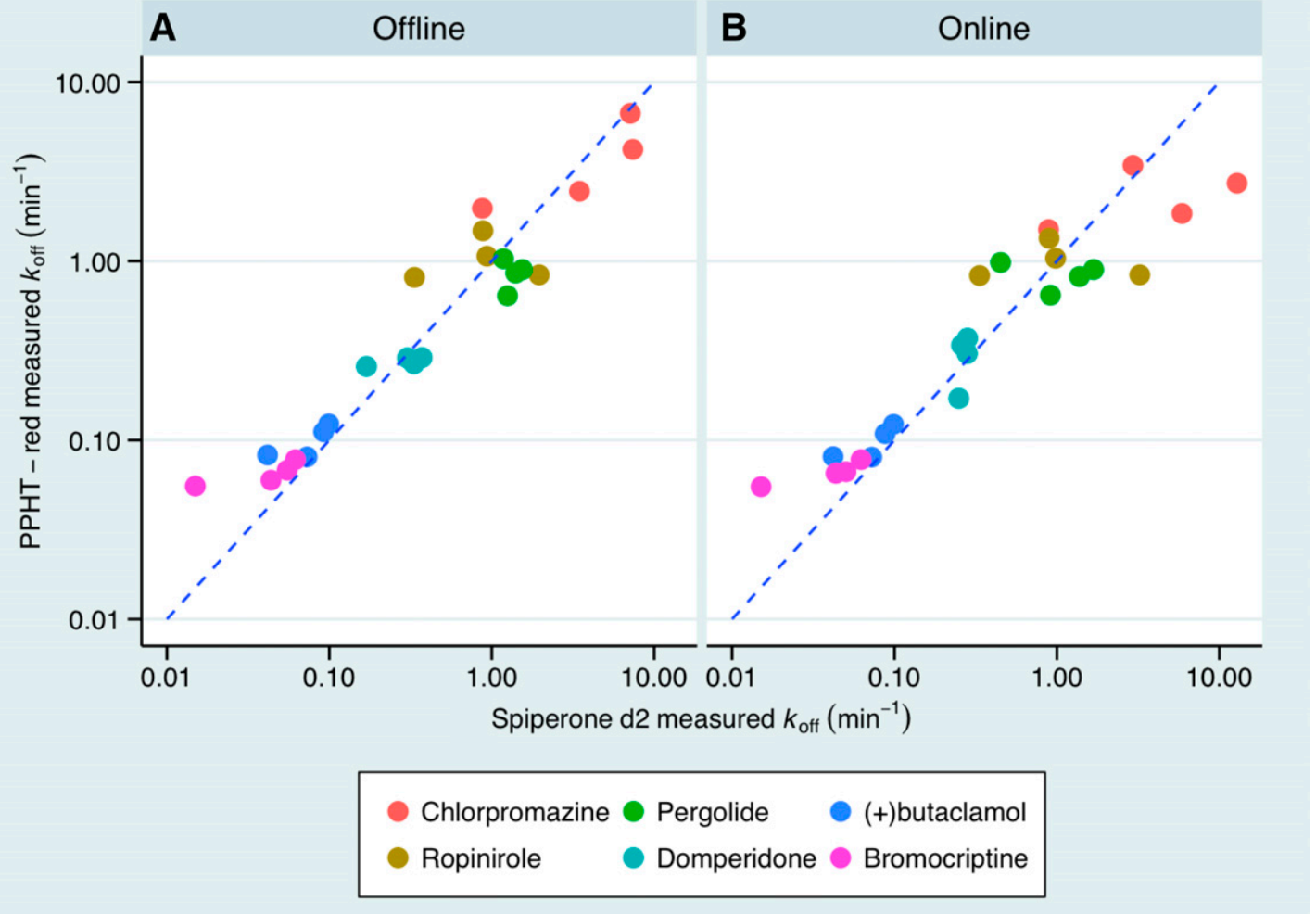

Fig. 9. Effect of tracer properties and assay configuration on the determination of unlabeled compound kinetic parameters at the human dopamine $\mathrm{D}_{2}$ receptor. Comparison of unlabeled compound $k_{\text {off }}$ values with determined using spiperone-d2 and PPHT-red following an off-line injection protocol with a 5 -second interval (A) and an on-line addition protocol with a 5 -second interval (B). CHO-D 2 R cell membranes $(2 \mu \mathrm{g} /$ well $)$ were incubated for 20 minutes with gentle agitation with a fixed concentration of PPHT-red or spiperone-d2 and increasing concentrations of competitor. Data are presented as individual estimates from four experiments

and D. Providing an initial start time of 1 second was used (representative of on-line injection), it was possible to determine accurately the kinetics of all model tracers with less than $10 \%$ variation around the mean. In contrast, employing a start time of 30 seconds prevented accurate kinetic determinations for the most rapidly dissociating tracer even when the read frequency was restricted to 1 second (Fig. 2, C and D), demonstrating the importance of on-line injection.

The situation with the Motulsky-Mahan eq. 2 for the estimation of unlabeled compound kinetics was a little more complicated. Although it was possible to derive accurate rate constant estimates for the majority of conditions, there were some combinations that failed to return reproducible estimates (Supplemental Tables 2-5). In general, employing a tracer concentration at $3 \times$, its $K_{\mathrm{d}}$ it was not possible to estimate reliably $k_{\text {on }}$ and $k_{\text {off }}$, where the dissociation of the tracer was ( $\geq 10$-fold) slower than the unlabeled competitor. For example, the accurate estimation of the rate constants of a rapidly dissociating ligand with $k_{\text {off }}$ of 100 minute $^{-1}$ requires a tracer that is also rapidly dissociating, i.e., in the range of 10 minute $^{-1}$. The initial read time was also critically important to determine the kinetics of unlabeled compounds. Where the simulations were started from 1 second, mimicking an online injection protocol, the kinetic parameters of unlabeled compounds were generally accurately estimated. In contrast, when measurements were started (sampled) 30 seconds after the beginning of the experiment, mimicking off-line addition, the estimates were poorer, particularly for rapidly dissociating ligands. This highlights the importance of early time points measured before equilibrium is established. This problem is exacerbated as read frequency is increased since definition on the tracer association curves are lost. In contrast, a short read frequency is associated with an increase in the number of data points, which is useful from an accuracy perspective (as illustrated by reduced \% CV values; see Supplemental Table 2). However, short read frequency will have a negative impact on throughput, which is an important consideration especially when profiling hundreds to thousands of compounds during screening.

To date, SPR has been the main method for measuring kinetics of fragments at receptors; however, this technique is traditionally limited to artificially stabilized receptors (Shepherd et al., 2014). Thus the competitive binding model presented is an attractive alternative to SPR and should theoretically allow the investigator to reveal the kinetics of lowaffinity fragments with off-rates in the order of 10 minute $^{-1}$ should an appropriate tracer be identified (see Supplemental Figs. 8 and 9).

In a previous paper exploring dopamine $\mathrm{D}_{2} \mathrm{R}$ agonist kinetics, we were able to demonstrate the importance of tracer properties on our ability to determine the kinetics of rapidly dissociating ligands (Klein-Herenbrink et al., 2016). In the current study, we have further explored this observation employing an on-line injection protocol. In the previous study, $\left[{ }^{3} \mathrm{H}\right]$-spiperone was unable to accurately determine the kinetics of the most rapidly dissociating $\mathrm{D}_{2} \mathrm{R}$ agonists. This is likely 
the result of a number of factors, including its slower measured off-rate from the dopamine $D_{2} R$, the relatively lower concentration of spiperone employed in the competition binding experiments, i.e., $3 \times\left[{ }^{3} \mathrm{H}\right]$-spiperone (versus $10 \times K_{\mathrm{d}}$ spiperone-d2 in the current study) and the decision to employ an initial start time of 30 seconds.

As predicted by the Monte Carlo simulations, PPHT-red, a tracer with a relatively fast off-rate, was more reliable at determining the kinetic off-rates of the most rapidly dissociating antagonists and agonists tested in this study. In contrast, spiperone-d2 $\left(10 \times K_{\mathrm{d}}\right)$, although adequate at determining the off-rates of the more slowly dissociating compounds, was prone to more variation in its determination of the off-rates of the most rapidly dissociating compounds. Importantly what this study clearly demonstrates is that a failure to demonstrate an accurate fit of the kinetics of a rapidly dissociating compound (10 minute $\left.{ }^{-1}\right)$ can be overcome through the use of higher concentrations of a slowly dissociating tracer and the decision to employ a shortening start and read frequency (see Fig. 7). As one might predict, the use of higher tracer concentrations could not compensate for an increase in the assay start time, representative of an offline addition protocol (see Supplemental Fig. 6).

What is apparent from this study is that for a competitive binding approach to be used throughout the different phases of the drug discovery process, then the kinetics of the tracer need to be tailored to the appropriate properties of the unlabeled compounds. For low-affinity compounds, such as initial hits or fragments, a rapidly equilibrating tracer is required to accurately determine rate constants. This is fortunate as it opens the possibility to fluorescently label an early hit to serve as a tracer to discover and characterize new fragments. In this format, it would be necessary to read relatively small numbers of wells in rapid succession (i.e., a shortened read frequency) using a repeat on-line injection protocol to resolve the kinetics of the most rapidly dissociating fragments. Also apparent is that as we move further along the drug discovery pathway toward lead optimization, it may be beneficial to label a more slowly dissociating compound, allowing the off-line addition of membranes and increased sample throughput through extended read frequency. The decision to employ shorter read times is ultimately a compromise between throughput and the accurate resolution of kinetic parameters.

In summary, we have improved the current understanding of the Motulsky-Mahan approach, providing clear guidelines on the use of tracers to measure the kinetics of unlabeled competitors. Based on the detailed Monte Carlo approach presented, we propose the following four factors as being important considerations when formulating competition association binding experiments:

1. Tracer kinetics: fast off tracers in the region of $0.1-1$ minute $^{-1}$ appear to be critical for the determination of unlabeled compounds with rapid off kinetics in the region of 1-10 minute ${ }^{-1}$.

2. Tracer concentration appears to play a crucial role in our ability to determine the kinetics of binding with greater accuracy and can even increase the range of compounds off rates that we can reliably measure.

3. Online injection capability proved critical to determine the off-rates of compounds and tracers that dissociate with off-rates in the region of 10 minute $^{-1}$.
4. Rapid read frequency dramatically improves the goodness of fit and reduces experimental variability and, like online injection, can rescue our ability to resolve the kinetics of the most rapidly dissociating compounds.

Experimental conditions can also be manipulated to enhance our ability to measure the kinetics of a particular tracer or competitor ligand. For example, reducing assay temperature will slow the off-rates of both the tracer and the competitor compound, a ploy that has been successfully used in the past to enable the determination of off-rates of more rapidly dissociating compounds using the off-line addition protocol (Contreras et al., 1986, Guo et al., 2012). It should be noted, however, that kinetic parameters calculated at lower temperatures are unlikely to reflect those in a physiologic system, significantly limiting their translational utility.

Overall, the findings in this paper highlight the importance of considering tracer kinetics and assay read start and read frequency when developing competition association assays. Notably, these simulations suggest that under the right conditions, the kinetic parameters of very low-affinity (millimolars) competitors can be measured, providing the opportunity for kinetic fragment-based receptor screens and the development of structure-kinetic relationships at all phases of the drug discovery cascade.

\section{Acknowledgments}

We thank Dr. Nick Holliday for his helpful suggestions during the preparation of this manuscript. In addition, we thank BMG Labtech Ltd. for their expert technical assistance and for personal sponsorship of David Sykes.

\section{Authorship Contributions}

Participated in research design: Sykes, Charlton.

Conducted experiments: Sykes.

Contributed new reagents or analytic tools: Jain.

Performed data analysis: Sykes, Jain.

Wrote or contributed to the writing of the manuscript: Sykes, Charlton.

\section{References}

Arányi P (1980) Kinetics of the hormone-receptor interaction. Competition experiments with slowly equilibrating ligands. Biochim Biophys Acta 628:220-227.

Bosma R, Stoddart LA, Georgi V, Bouzo-Lorenzo M, Bushby N, Inkoom L, Waring MJ, Briddon SJ, Vischer HF, Sheppard RJ, et al. (2019) Probe dependency in the determination of ligand binding kinetics at a prototypical $\mathrm{G}$ protein-coupled receptor. Sci Rep 9:7906.

Bouzo-Lorenzo M, Stoddart LA, Xia L, IJzerman AP, Heitman LH, Briddon SJ, and Hill SJ (2019) A live cell NanoBRET binding assay allows the study of ligandbinding kinetics to the adenosine $\mathrm{A}_{3}$ receptor. Purinergic Signal 15:139.

Contreras ML, Wolfe BB, and Molinoff PB (1986) Kinetic analysis of the interactions of agonists and antagonists with beta adrenergic receptors. J Pharmacol Exp Ther 239:136-143.

Carter CM, Leighton-Davies JR, and Charlton SJ (2007) Miniaturized receptor binding assays: complications arising from ligand depletion. J Biomol Screen 12: $255-266$.

Christopoulos A (2001) From 'captive' agonism to insurmountable antagonism: demonstrating the power of analytical pharmacology. Clin Exp Pharmacol Physiol 28:223-229.

Copeland RA (2016) The drug-target residence time model: a 10-year retrospective. Nat Rev Drug Discov 15:87-95.

Dowling MR and Charlton SJ (2006) Quantifying the association and dissociation rates of unlabelled antagonists at the muscarinic M3 receptor. $\mathrm{Br} J$ Pharmacol 148:927-937.

Emami-Nemini A, Roux T, Leblay M, Bourrier E, Lamarque L, Trinquet E, and Lohse MJ (2013) Time-resolved fluorescence ligand binding for G proteincoupled receptors. Nat Protoc 8:1307-1320.

Fleck BA, Hoare SR, Pick RR, Bradbury MJ, and Grigoriadis DE (2012) Binding kinetics redefine the antagonist pharmacology of the corticotropin-releasing factor type 1 receptor. J Pharmacol Exp Ther 341:518-531.

Georgi V, Schiele F, Berger BT, Steffen A, Marin Zapata PA, Briem H, Menz S, Preusse C, Vasta JD, Robers MB, et al. (2018) Binding kinetics survey of the drugged kinome. J Am Chem Soc 140:15774-15782. 
Gillard M, Van Der Perren C, Moguilevsky N, Massingham R, and Chatelain P (2002) Binding characteristics of cetirizine and levocetirizine to human $\mathrm{H}(1)$ histamine receptors: contribution of Lys(191) and Thr(194). Mol Pharmacol 61:391-399.

Gillard M and Chatelain P (2006) Changes in $\mathrm{pH}$ differently affect the binding properties of histamine H1 receptor antagonists. Eur J Pharmacol 530 (3): 205-214, doi: 10.1016/j.ejphar.2005.11.051 16388798

Guo D, Heitman LH, and IJzerman AP (2017) Kinetic aspects of the interaction between ligand and $\mathrm{G}$ protein-coupled receptor: the case of the adenosine receptors. Chem Rev 117:38-66

Guo D, Mulder-Krieger T, IJzerman AP, and Heitman LH (2012) Functional efficacy of adenosine $\mathrm{A}_{2} \mathrm{~A}$ receptor agonists is positively correlated to their receptor residence time. $\mathrm{Br}$ J Pharmacol 166:1846-1859.

Klein Herenbrink C, Sykes DA, Donthamsetti P, Canals M, Coudrat T, Shonberg J, Scammells PJ, Capuano B, Sexton PM, Charlton SJ, et al. (2016) The role of kinetic context in apparent biased agonism at GPCRs. Nat Commun 7:10842.

Martella A, Sijben H, Rufer AC, Grether U, Fingerle J, Ullmer C, Hartung T, IJzerman AP, van der Stelt M, and Heitman LH (2017) A novel selective inverse agonist of the $\mathrm{CB}_{2}$ receptor as a radiolabeled tool compound for kinetic binding studies. Mol Pharmacol 92:389-400.

Motulsky HJ and Mahan LC (1984) The kinetics of competitive radioligand binding predicted by the law of mass action. Mol Pharmacol 25:1-9.

Motulsky HJ and Mahan LC (1984) The kinetics of competitive radioligand binding predicted by the law of mass action. Mol Pharmacol 25 (1):1-9.

Nederpelt I, Georgi V, Schiele F, Nowak-Reppel K, Fernández-Montalván AE, IJzerman AP, and Heitman LH (2016) Characterization of $12 \mathrm{GnRH}$ peptide agonists - a kinetic perspective. Br J Pharmacol 173:128-141.

Riddy DM, Valant C, Rueda P, Charman WN, Sexton PM, Summers RJ, Christopoulos A, and Langmead CJ (2015) Label-free kinetics: exploiting functional hemiequilibrium to derive rate constants for muscarinic receptor antagonists. $\mathrm{Mol}$ Pharmacol 88:779-790.

Schiele F, Ayaz P, and Fernández-Montalván A (2015) A universal homogeneous assay for high-throughput determination of binding kinetics [published correction appears in Anal Biochem (2016) 514:1]. Anal Biochem 468:42-49.

Schmidtke P, Luque FJ, Murray JB, and Barril X (2011) Shielded hydrogen bonds as structural determinants of binding kinetics: application in drug design. J Am Chem Soc 133:18903-18910.

Shepherd CA, Hopkins AL, and Navratilova I (2014) Fragment screening by SPR and advanced application to GPCRs. Prog Biophys Mol Biol 116:113-123.

Slack RJ, Russell LJ, Hall DA, Luttmann MA, Ford AJ, Saunders KA, Hodgson ST, Connor HE, Browning C, and Clark KL (2011) Pharmacological characterization of GSK1004723, a novel, long-acting antagonist at histamine $\mathrm{H}(1)$ and $\mathrm{H}(3)$ receptors Br J Pharmacol 164:1627-1641.

Stoddart LA, Johnstone EKM, Wheal AJ, Goulding J, Robers MB, Machleidt T, Wood $\mathrm{KV}$, Hill SJ, and Pfleger KDG (2015) Application of BRET to monitor ligand binding to GPCRs. Nat Methods 12:661-663.

Stoddart LA, Vernall AJ, Bouzo-Lorenzo M, Bosma R, Kooistra AJ, de Graaf C Vischer HF, Leurs R, Briddon SJ, Kellam B, et al. (2018) Development of novel fluorescent histamine $\mathrm{H}_{1}$-receptor antagonists to study ligand-binding kinetics in living cells. Sci Rep 8:1572.
Sullivan SK, Hoare SR, Fleck BA, Zhu YF, Heise CE, Struthers RS, and Crowe PD (2006) Kinetics of nonpeptide antagonist binding to the human gonadotropinreleasing hormone receptor: implications for structure-activity relationships and insurmountable antagonism. Biochem Pharmacol 72:838-849.

Sykes DA, Bradley ME, Riddy DM, Willard E, Reilly J, Miah A, Bauer C, Watson SJ, Sandham DA, Dubois G, et al. (2016) Fevipiprant (QAW039), a slowly dissociating CRTh2 antagonist with the potential for improved clinical efficacy. Mol Pharmacol 89:593-605.

Sykes DA and Charlton SJ (2012) Slow receptor dissociation is not a key factor in the duration of action of inhaled long-acting $\beta 2$-adrenoceptor agonists. Br J Pharmacol 165:2672-2683.

Sykes DA and Charlton SJ (2018) Single Step Determination of Unlabeled Compound Kinetics Using a Competition Association Binding Method Employing TimeResolved FRET. Methods Mol Biol 1824:177-194.

Sykes DA, Dowling MR, and Charlton SJ (2009) Exploring the mechanism of agonist efficacy: a relationship between efficacy and agonist dissociation rate at the muscarinic M3 receptor. Mol Pharmacol 76:543-551.

Sykes DA, Dowling MR, and Charlton SJ (2010) Measuring receptor target coverage: a radioligand competition binding protocol for assessing the association and dissociation rates of unlabeled compounds, Curr Protoc Pharmacol Chapter 9, p Unit 9.14

Sykes DA, Lane JR, Szabo M, Capuano B, Javitch JA, and Charlton SJ (2018) Reply to 'Antipsychotics with similar association kinetics at dopamine $\mathrm{D}_{2}$ receptors differ in extrapyramidal side-effects'. Nat Commun 9:3568.

Sykes DA, Moore H, Stott L, Holliday N, Javitch JA, Lane JR, and Charlton SJ (2017) Extrapyramidal side effects of antipsychotics are linked to their association kinetics at dopamine $\mathrm{D}_{2}$ receptors. Nat Commun 8:763.

Sykes DA, Parry C, Reilly J, Wright P, Fairhurst RA, and Charlton SJ (2014) Observed drug-receptor association rates are governed by membrane affinity: the importance of establishing "micro-pharmacokinetic/pharmacodynamic relationships" at the $\beta 2$-adrenoceptor. Mol Pharmacol 85:608-617.

Turner RJ and Charlton SJ (2005) Assessing the minimum number of data points required for accurate IC50 determination. Assay Drug Dev Technol 3:525-531.

Yu Z, IJzerman AP, and Heitman LH (2015) Kv 11.1 (hERG)-induced cardiotoxicity: a molecular insight from a binding kinetics study of prototypical Kv 11.1 (hERG) inhibitors. Br J Pharmacol 172:940-955.

Vauquelin G (2016) Effects of target binding kinetics on in vivo drug efficacy: koff, kon and rebinding. $\mathrm{Br} J$ Pharmacol 173:2319-2334.

Xia L, de Vries H, IJzerman AP, and Heitman LH (2016) Scintillation proximity assay (SPA) as a new approach to determine a ligand's kinetic profile. A case in point for the adenosine A1 receptor. Purinergic Signal 12:115-126.

Address correspondence to: Steven J. Charlton, School of Life Sciences, Queen's Medical Centre, University of Nottingham, Nottingham NG7 2UH, UK. E-mail: steven.charlton@nottingham.ac.uk; David A. Sykes, School of Life Sciences, Queen's Medical Centre, University of Nottingham, Nottingham NG7 2UH, UK. E-mail: David.Sykes@nottingham.ac.uk 